\title{
Robust Adaptive Stabilization of Linear Time-Invariant Dynamic Systems by Using Fractional-Order Holds and Multirate Sampling Controls
}

\author{
S. Alonso-Quesada and M. De la Sen \\ Department of Electricity and Electronics, Faculty of Science and Technology, \\ University of Basque Country, Leioa 48940, Spain \\ Correspondence should be addressed to S. Alonso-Quesada, santi@we.lc.ehu.es \\ Received 8 June 2009; Accepted 2 February 2010 \\ Academic Editor: Francisco Solis
}

Copyright (c) 2010 S. Alonso-Quesada and M. De la Sen. This is an open access article distributed under the Creative Commons Attribution License, which permits unrestricted use, distribution, and reproduction in any medium, provided the original work is properly cited.

\begin{abstract}
This paper presents a strategy for designing a robust discrete-time adaptive controller for stabilizing linear time-invariant (LTI) continuous-time dynamic systems. Such systems may be unstable and noninversely stable in the worst case. A reduced-order model is considered to design the adaptive controller. The control design is based on the discretization of the system with the use of a multirate sampling device with fast-sampled control signal. A suitable on-line adaptation of the multirate gains guarantees the stability of the inverse of the discretized estimated model, which is used to parameterize the adaptive controller. A dead zone is included in the parameters estimation algorithm for robustness purposes under the presence of unmodeled dynamics in the controlled dynamic system. The adaptive controller guarantees the boundedness of the system measured signal for all time. Some examples illustrate the efficacy of this control strategy.
\end{abstract}

\section{Introduction}

Adaptive control theory has been widely applied for stabilizing increasingly complex engineering systems with large uncertainties [1], including the incorporation of parallel multiestimation and time-delayed and hybrid models [2-6]. Such model uncertainties may come from the fact that the parameters of the dynamic system model are partially or fully unknown and/or from the presence of unmodeled dynamics [3]. On the other hand, discrete equations are useful for modeling and controlling discretized continuous-time systems in practical situations $[2,5-9]$ and as a tool for describing more complex nonlinear structures via discretization $[10,11]$. A frequently used method to stabilize an unknown dynamic system is 
based on the model reference adaptive control (MRAC) problem [12]. However, the presence of unstable system zeros does not make possible the design of a controller to achieve the model-matching objective unless such unstable zeros are transmitted to the reference model $[1,2,6,13,14]$. Unfortunately, such a transmission cannot be available if some of the zeros of the system to be controlled are unknown as it can occur in the context of the adaptive control where the dynamic system may be completely or partially unknown. However, there are several alternative methods to circumvent this difficulty and carry out the stable adaptive control design. Two of them depend on relaxing the control objective from the MRAC to the less exigent adaptive pole-placement control (APPC) $[15,16]$. In this way, the stabilization of the feedback (or closed-loop) system can be ensured although its transient behavior cannot be fixed to a predefined one. On one hand, the method in [15] includes a modification in the estimated parameters to ensure the controllability of the estimation model of the dynamic system. In this way, closed-loop unstable pole-zero cancellations are avoided, which is crucial for the controller synthesis. Such a method is applicable for both continuous-time and discrete-time dynamic systems to be controlled. On the other hand, the research [16] may be used in the case of continuous-time dynamic systems. There, a periodic piecewise constantgain controller is added in the feedback chain. In the nonadaptive case, the gain values are those required so that the discretized system model under the fundamental sampling period and a zero-order hold $(\mathrm{ZOH})$ could be stabilized. It is worth that such a control gain is piecewise constant during the sampling period in order to place the discretized poles at stable desired locations. Concretely, each sampling period is split into a certain finite number of uniform subintervals and the control gain takes a different value within each subinterval. In this way, the controller consists of a constant vector of gains. In this sense, the controller works with a sampling rate faster than that used to discretize the controlled system. In the adaptive case, the discretized dynamic system model parameters are on-line updated; then the controller gains vector is time varying and converges asymptotically to a constant one.

Another method, which does not relax the MRAC objective, to overcome the drawback of the unstable zeros of a continuous-time dynamic system is the design of a discrete-time controller with the use of a hold device combined with a multirate sampling with fast input rate in the discretization of the continuous-time system [7, 8]. In this way, an inversely stable estimated model of the discretized dynamic system can be obtained and a controller can be designed to match a stable arbitrarily chosen discrete-time reference model since all of the discretized zeros may be cancelled if suited. In this context, this paper presents a robust adaptive control scheme for stabilizing uncertain controlled continuous-time dynamic systems while matching a freely chosen discrete-time reference model, with a bounded tracking-error, to be applied when the continuous-time system is unknown and subject to the presence of unmodeled dynamics. The main novelty is that the discrete zeros may be always stabilized even if the zeros of the continuous system are not all stable. As a result, the reference model zeros can all be freely fixed. The control scheme is based on the discretization process by combining the use of a fractionalorder hold $(\mathrm{FROH})$ and a multirate with fast sampling control signal. In this way, the estimated discretized model can be guaranteed to be inversely stable by means of a suitable on-line updating of the multirate gains without requirements neither on the stability of the continuous-time zeros nor the size of the sampling period [17]. Such a strategy gives place to hybrid systems where continuous-time and discrete-time dynamics are mixed. Concretely, a discrete-time controller is designed to govern the behavior of a continuous-time dynamical system. Epidemic control of infectious diseases and species population control systems in Ecology are two typical examples of this class of hybrid systems [18-20]. A discrete-time control strategy depending on a pulse vaccination is designed in [18]. Such vaccination pulses 
work as a discrete-time control signal and the eradication of the diseases is reached provided that the vaccination rate is sufficiently large. The researches $[19,20]$ study, respectively, the necessary conditions which guarantee the stabilization and permanence of single-species and predator-prey systems populations in their respective habitats. Both are continuoustime dynamic systems which can be described efficiently by means of discrete-time models in order to prescribe its evolution and then to develop discrete-time control strategies to ensure the permanence of the species. All of these systems are subject to eventual changes in the continuous-time dynamics whenever the discrete-time control action takes place. In this sense, they belong to a class of switched systems whose stability and stabilization conditions have been studied in the recent literature $[4,21]$.

Furthermore, the presented control strategy guarantees the stabilization of the continuous-time dynamic system without any assumption about the stability of its zeros, which had been supposed in previous works [13, 22], and without requiring estimates modification in contrast with previous works on the subject $[2,15]$. Such relaxations constitute the main contribution of the present work. A FROH is used since it allows a better accommodation of discrete adaptive techniques to the transient response of discretetime controlled continuous-time dynamic systems [9]. Furthermore, the estimation algorithm includes a relative adaptation dead-zone to deal with the presence of unmodeled dynamics [14]. Such a dead-zone is crucial to ensure the estimates convergence and the stability of the adaptive control system. The stabilization is guaranteed provided that (1) the continuous-time dynamic system is stabilizable and observable (2) the size of the unmodeled dynamics is sufficiently small, and (3) such an unmodeled dynamics can be related to the system input by means of additive and/or multiplicative stable transfer functions.

The paper is organized as follows. Section 2 presents the discretization process used to obtain an inversely stable discretized dynamic system model from a possibly inversely unstable continuous-time dynamic system. Section 3 deals with the control design to match a discrete-time reference model at sampling instants for both nonadaptive and adaptive cases. Then, the stability analysis of the designed adaptive control algorithm is presented in Section 4. Finally, simulation results, which illustrate the behavior of the adaptive control system, are shown in Section 5 and conclusions end the paper in Section 6.

\section{Problem Statement}

Consider a linear time invariant SISO and strictly proper continuous-time dynamic system described by the following state-space equations:

$$
\dot{x}(t)=A x(t)+B u(t), \quad y(t)=C x(t),
$$

where $u(t)$ and $y(t)$ are, respectively, the control (or input) and measured (or output) signals, $x(t) \in \mathbb{R}^{n}$ is the state vector, and $A, B$, and $C$ are constant matrices of suitable dimensions. The transfer function of $(2.1)$ is $G_{p}(s)=q(s) / p(s)=C\left(s I_{n}-A\right)^{-1} B$ where $n=\operatorname{Deg}(p(s)) \geq$ $\operatorname{Deg}(q(s))=m, s$ denotes the Laplace transform argument [1], and $I_{n}$ represents the $n$-order identity matrix. In the sequel, the controlled dynamic system is referred to as the "plant" to be controlled as it is commonly referred to in the Engineering Automation context. The following assumptions are made on the plant. 
Assumption 1. (i) An upper-bound $\bar{n}$ of the plant order $n$ is known as it is the nominal order $n_{0} \leq n$.

(ii) The plant realization matrices can be expressed as

$$
A=\left[\begin{array}{c|c}
A_{0} & 0 \\
\hline A_{21} & A_{22}
\end{array}\right], \quad B=\left[\begin{array}{c}
B_{0} \\
\hline B_{21}
\end{array}\right], \quad C=\left[C_{0} \mid C_{12}\right],
$$

where $\left\{A_{0}, B_{0}, C_{0}\right\}$ denotes the state-space realization for the nominal model of the plant and the other matrix blocks are related to the unmodeled dynamics. In this context, the state vector is composed of two sets of state variables, namely, $x \triangleq\left[x_{0}^{T} \mid x_{1}^{T}\right]^{T}$ where $x_{0} \in \mathbb{R}^{n_{0}}$ is the nominal state vector. Furthermore, the eigenvalues of the block $A_{22}$ are strictly stable, $\left\|B_{21}\right\| \leq \mu_{0}$, and $\left\|C_{12}\right\| \leq \mu_{0}$ for some sufficiently small real $\mu_{0}>0$ with $\|M\|$ denoting the norm of the matrix $M$.

(iii) The nominal pair $\left(A_{0}, C_{0}\right)$ is observable.

Remark 2.1. (i) Given any state-space realization, there always exists an infinite number of state-space realizations in triangular form as (2.2) which have the same transfer function that the former has. Each one of such state-space realizations may be obtained by applying an appropriate coordinates transformation on the original realization. Then, whenever the original state-space realization of the plant is not a triangular form, an appropriate coordinates transformation is required to obtain a triangular form state-space realization as (2.2).

(ii) The internal representation (2.2) gives place to an input-output relation defined by a transfer function as

$$
G_{p}(s)=G_{0}(s)\left(1+\Delta_{m}(s)\right)+\Delta_{a}(s)
$$

where $G_{0}(s)$ denotes the transfer function of the plant nominal model and $\Delta_{m}(s)$ and $\Delta_{a}(s)$ are two rational transfer functions related to the multiplicative and additive unmodeled dynamics, respectively. The poles of $\Delta_{m}(s)$ and $\Delta_{a}(s)$ are the eigenvalues of the block $A_{22}$ and their gains depend on the norms of the blocks $B_{21}$ and $C_{12}$.

(iii) If the nominal triple $\left\{A_{0}, B_{0}, C_{0}\right\}$ is known, then a classical pole-placement controller may be synthesized without using estimation. However, this knowledge is not necessary to synthesize adaptive control with the less restrictive knowledge of $n_{0}$, which is the nominal plant order.

The plant can be unstable and of nonstable inverse. Then, the use of the modelmatching technique, with a free-chosen reference model, for the controller synthesis can be used with a discrete-time controller. In such a case, the reconstruction process of the continuous-time plant input from the discrete-time control output gives the possibility of obtaining an inversely stable discretized plant model. Such a reconstruction has to be made with the use of a hold device, a FROH in the most general case, combined with a multirate with fast input rate. This multirate provides free-design parameters, related to multirate gains, which can be adjusted so that the discretized plant model could be of stable inverse. In this way, the model-matching technique can be used to synthesize a discrete-time controller 
to stabilize the continuous-time plant while matching a freely chosen reference model at sampling instants. The plant input obtained from such a reconstruction method is given by

$$
u(t)=\alpha_{j}\left\{u(k)+\beta \frac{u(k)-u(k-1)}{T}(t-k T)\right\}
$$

for $t \in\left[k T+(j-1) T^{\prime}, k T+j T^{\prime}\right), j \in\{1,2, \ldots, N\}$, where $\beta \in[-1,1] \cap \mathbb{R}$ is the $\mathrm{FROH}$ correcting gain, $T$ is the sampling period for the state and output (slow sampling) which is uniformly divided in $N$ subperiods of length $T^{\prime}=T / N$ (fast sampling) to generate the fast sampling plant input, $u(k)$ denotes the value of the controller output sequence at the instant $k T$, for all $k \in \mathbb{Z}_{0}^{+} \triangleq \mathbb{Z}^{+} \cup\{0\}$, and $\alpha_{j} \in \mathbb{R}$ are the multirate gains. The technique of using $T \neq T^{\prime}<T$, with $N$ exceeding some prescribed lower bound, is the key feature for always achieving a stable discrete-time transfer function numerator even if the continuous-time plant transfer function $G_{p}(s)$ has some critically stable or unstable zero. In this sense, the FROH device operates on the sequence $\{u(k)\}$ defined at the slow sampling instants $k T$ and then the input $u(t)$ is generated over each subperiod $T^{\prime}$ with the corresponding gain $\alpha_{j}$. Such gains have to be suitably selected to ensure the stability of the zeros of the discretized plant model which relates the sequences $\{u(k)\}$ and $\{y(k)\}$ (plant output sequence) defined over the sampling period $T$.

The state-space representation corresponding to the discrete-time plant obtained from the discretization of the continuous-time plant by applying the $\mathrm{FROH}$ with the multirate is given by

$$
x_{0}(k+1)=F(T) x_{0}(k)+H_{1}(T) u(k)+H_{2}(T) u(k-1), \quad y(k)=C_{0} x_{0}(k)+\eta(k),
$$

where $\eta(k)$ denotes the contribution of the unmodeled dynamics to the discretized plant output, $F(T)=\psi^{N}(T)=\phi(T)=e^{A_{0} T} \in \mathbb{R}^{n_{0} \times n_{0}}$ is the state transition matrix of the continuoustime nominal dynamic system valued during a sampling period, and

$$
\begin{gathered}
H_{1}(T)=\sum_{\ell=1}^{N} \alpha_{\ell} \psi^{N-\ell}(T)\left[\left(1+\frac{\ell-1}{N} \beta\right) \Gamma\left(T^{\prime}\right)+\frac{\beta}{T} \Gamma^{\prime}\left(T^{\prime}\right)\right]=C_{\Delta}(T) g \in \mathbb{R}^{n_{0} \times 1}, \\
H_{2}(T)=-\beta \sum_{\ell=1}^{N} \alpha_{\ell} \psi^{N-\ell}(T)\left[\frac{\ell-1}{N} \Gamma\left(T^{\prime}\right)+\frac{1}{T} \Gamma^{\prime}\left(T^{\prime}\right)\right]=-\beta C_{\Delta}^{\prime}(T) g \in \mathbb{R}^{n_{0} \times 1},
\end{gathered}
$$


with

$$
\begin{aligned}
& \Gamma\left(T^{\prime}\right)=\int_{0}^{T^{\prime}} \phi\left(T^{\prime}-s\right) B_{0} d s \in \mathbb{R}^{n_{0} \times 1}, \\
& C_{\Delta}(T)=\left[\begin{array}{llll}
\psi^{N-1}(T) \Delta_{1}(T) & \cdots & \psi(T) \Delta_{N-1}(T) & \Delta_{N}(T)
\end{array}\right] \in \mathbb{R}^{n_{0} \times N}, \\
& \Gamma^{\prime}\left(T^{\prime}\right)=\int_{0}^{T^{\prime}} \phi\left(T^{\prime}-s\right) B_{0} s d s \in \mathbb{R}^{n_{0} \times 1}, \\
& C_{\Delta}^{\prime}(T)=\left[\begin{array}{llll}
\psi^{N-1}(T) \Delta_{1}^{\prime}(T) & \cdots & \psi(T) \Delta_{N-1}^{\prime}(T) & \Delta_{N}^{\prime}(T)
\end{array}\right] \in \mathbb{R}^{n_{0} \times N}, \\
& \Delta_{j}(T)=\left(1+\frac{j-1}{N} \beta\right) \Gamma\left(T^{\prime}\right)+\frac{\beta}{T} \Gamma^{\prime}\left(T^{\prime}\right) \in \mathbb{R}^{n_{0} \times 1}, \\
& \Delta_{j}^{\prime}(T)=\frac{j-1}{N} \Gamma\left(T^{\prime}\right)+\frac{1}{T} \Gamma^{\prime}\left(T^{\prime}\right) \in \mathbb{R}^{n_{0} \times 1}, \quad g=\left[\alpha_{1} \cdots \alpha_{N}\right]^{T} .
\end{aligned}
$$

\subsection{Input-Output Relation for the Discretized Plant}

From the output equation of (2.5), substituting the state-space equation and iterating $n_{0}$ times, it follows that

$y(k)$

$$
\begin{aligned}
= & C_{0}\left\{F^{n_{0}} x_{0}\left(k-n_{0}\right)+H_{1} u(k-1)+\sum_{i=1}^{n_{0}-1} F^{i-1}\left(F H_{1}+H_{2}\right) u(k-i-1)+F^{n_{0}-1} H_{2} u\left(k-n_{0}-1\right)\right\} \\
& +\eta(k),
\end{aligned}
$$

where the argument $T$ in $F(T), H_{1}(T)$, and $H_{2}(T)$ has been omitted for simplicity. In a similar way, one can obtain that

$$
\begin{aligned}
& y(k-\ell) \\
& =C_{0}\left\{F^{n_{0}-\ell} x_{0}\left(k-n_{0}\right)+H_{1} u(k-\ell-1)+\sum_{i=\ell+1}^{n_{0}-1} F^{i-\ell-1}\left(F H_{1}+H_{2}\right) u(k-i-1)\right. \\
& \left.\quad+F^{n_{0}-\ell-1} H_{2} u\left(k-n_{0}-1\right)\right\}+\eta(k-\ell)
\end{aligned}
$$

for $\ell \in\left\{1,2, \ldots, n_{0}-1\right\}$. (2.9) together with $y\left(k-n_{0}\right)=C_{0} x_{0}\left(k-n_{0}\right)+\eta\left(k-n_{0}\right)$ can be rewritten as

$$
Y_{v}(k-1)=V x_{0}\left(k-n_{0}\right)+\Pi_{v}(k-1)+\Phi U_{v}(k-2),
$$


where

$$
\begin{gathered}
Y_{v}(k-1)=\left[\begin{array}{lllcc}
y\left(k-n_{0}\right) & y\left(k-n_{0}+1\right) & \cdots & y(k-2) & y(k-1)
\end{array}\right]^{T}, \\
V=\left[\begin{array}{lllll}
C_{0}^{T} & \left(C_{0} F\right)^{T} & \cdots & \left(C_{0} F^{n_{0}-2}\right)^{T} & \left(C_{0} F^{n_{0}-1}\right)^{T}
\end{array}\right]^{T}, \\
\Pi_{v}(k-1)=\left[\begin{array}{lllcc}
\eta\left(k-n_{0}\right) & \eta\left(k-n_{0}+1\right) & \cdots & \eta(k-2) & \eta(k-1)
\end{array}\right]^{T}, \\
U_{v}(k-2)=\left[\begin{array}{lllll}
u\left(k-n_{0}-1\right) & u\left(k-n_{0}\right) & \cdots & u(k-3) & u(k-2)
\end{array}\right]^{T}, \\
\Phi=\left[\begin{array}{ccccc}
0 & 0 & \cdots & 0 & 0 \\
C_{0} H_{2} & C_{0} H_{1} & \cdots & 0 & 0 \\
C_{0} F H_{2} & C_{0}\left(F H_{1}+H_{2}\right) & \cdots & 0 & 0 \\
\vdots & \vdots & \ddots & \vdots & \vdots \\
C_{0} F^{n_{0}-3} H_{2} & C_{0} F^{n_{0}-4}\left(F H_{1}+H_{2}\right) & \cdots & C_{0} H_{1} & 0 \\
C_{0} F^{n_{0}-2} H_{2} & C_{0} F^{n_{0}-3}\left(F H_{1}+H_{2}\right) & \cdots & C_{0}\left(F H_{1}+H_{2}\right) & C_{0} H_{1}
\end{array}\right]
\end{gathered}
$$

with $V$ being the observability matrix for the discretized plant nominal model. By substituting the expression for $x_{0}\left(k-n_{0}\right)$, obtained from (2.10), in (2.8) it follows that

$$
y(k)=-\sum_{i=1}^{n_{0}} a_{i} y(k-i)+\sum_{i=1}^{n_{d}} b_{i} u(k-i)+\Omega(k)=-\sum_{i=1}^{n_{0}} a_{i} y(k-i)+\sum_{i=1}^{n_{d}} \sum_{j=1}^{N} \alpha_{j} b_{i, j} u(k-i)+\Omega(k)
$$

where $n_{d}=n_{0}$ if $\beta=0$ or $n_{d}=n_{0}+1$ if $\beta \neq 0$, and

$$
\begin{aligned}
a_{i} & =-\left[C_{0} F^{n_{0}} V^{-1}\right]_{n_{0}-i+1^{\prime}} \\
b_{i} & =\sum_{j=1}^{N} \alpha_{j} b_{i, j}=C_{0} F^{i-2}\left(F H_{1}+H_{2}\right)-\left[C_{0} F^{n_{0}} V^{-1} \Phi\right]_{n_{0}-i+2} \text { for } i \in\left\{2,3, \ldots, n_{0}\right\}, \\
b_{1} & =\sum_{j=1}^{N} \alpha_{j} b_{1, j}=C_{0} H_{1}, \quad b_{n_{0}+1}=\sum_{j=1}^{N} \alpha_{j} b_{n_{0}+1, j}=C_{0} F^{n_{0}-1} H_{2}-\left[C_{0} F^{n_{0}} V^{-1} \Phi\right]_{1}, \\
\Omega(k) & =\eta(k)-\sum_{i=1}^{n_{0}}\left[C_{0} F^{n_{0}} V^{-1}\right]_{n_{0}-i+1} \eta(k-i)
\end{aligned}
$$

with $[v]_{i}$ denoting the $i$ th component of the vector $v$ and having into account the expressions (2.6) for $H_{1}(T)$ and $H_{2}(T)$. Note that $\Omega(k)$ contains the contribution of the unmodeled dynamics to the discretized plant model output.

Remark 2.2. (i) The observability of the pair $\left(A_{0}, C_{0}\right)$ implies the nonsingularity of $V$ whenever $T \geq T_{0}>0$, for some sufficiently small real $T_{0}$, and conversely. Note that $V$ tends 
to be singular as the sampling period $T$ tends to zero since $F(T)$ would tend to the identity matrix.

(ii) The modeled part of the discretized plant (2.12) can be expressed equivalently as the discrete transfer function

$$
G_{d}(z)=\frac{B_{d}(z)}{A_{d}(z)}
$$

where $A_{d}(z)=z^{n_{0}+1}+\sum_{i=1}^{n_{0}} a_{i} z^{n_{0}-i+1}$ and $B_{d}(z)=\sum_{i=1}^{n_{0}+1} b_{i} z^{n_{0}-i+1}$ with $z$ being the Z-transform argument used in discrete-time transfer functions [23]. In the particular case that $\beta=0$ it follows from (2.6) that $H_{2}(T)=0$, then $b_{n_{0}+1}=0$ since the first column of $\Phi$ is zero and then the transfer function $G_{d}(z)$ possesses a zero-pole cancellation at $z=0$; that is, its order is $n_{0}$ instead of $n_{0}+1$. In the rest of the paper, $n_{d} \in \mathbb{Z}^{+}$is used to denote the order of such a transfer function being $n_{d}=n_{0}$ if $\beta=0$ (i.e., a $\mathrm{ZOH}$ is used in the discretization process) or $n_{d}=n_{0}+1$ if $\beta \neq 0$. The parameter $n_{d}$ allows a unified definition of polynomials and discrete transfer functions for different degrees associated with $\beta=0$ and $\beta \neq 0$.

Note that the coefficients $b_{i}$, for $i \in\left\{1,2, \ldots, n_{d}\right\}$, of the polynomial $B_{d}(z)$ depend on the multirate gains $\alpha_{j}$, for $j \in\{1, \ldots, N\}$, included in $H_{1}(T)$ and $H_{2}(T)$; that is,

$$
v=M g
$$

where $v=\left[\begin{array}{llll}b_{1} & b_{2} & \cdots & b_{n_{d}}\end{array}\right]^{T}$ and $M=\left[b_{i, j}\right] \in \mathbb{R}^{n_{d} \times N}$. The components $b_{i, j}$ depend on the sampling period $T$, the correcting gain $\beta \in[-1,1]$ of the $\mathrm{FROH}$, and the matrices $A_{0}, B_{0}$ and $C_{0}$ which define the plant nominal model. In this context, if the multirate gain vector is suitably chosen, one may fix the coefficients $b_{i}$ at desired values and, in this way, one may place the zeros of the discretized plant nominal model at desired locations, namely, within the stability domain. This is the strategy to get the stabilization of the closed-loop system by means of a model-matching controller.

Assumption 2. The correcting gain $\beta$ of the $\mathrm{FROH}$ and the sampling period $T$ are chosen such that $M$ is a full-rank matrix.

Remark 2.3. In the non-adaptive case (known plant parameters), Assumption 2 is crucial to calculate the multirate parameterization $g$ from (2.15) provided that $N \geq n_{d}$. If $N=n_{d}$, $g=M^{-1} v$ is the unique solution for the multirate gains which places the discretized plant zeros at prefixed locations, those linked to the roots of the polynomial whose coefficients are the components of $v$. In this way, if the zeros of such a polynomial are within the stability domain, then the discretized plant transfer function is inversely stable. On the contrary, if $N>n_{d}$, different solutions can be obtained for $g$. However, in the adaptive case, such an assumption can be relaxed if the parameters estimation algorithm guarantees that the rank of the matrix $\widehat{M}(k)$, composed with the estimates of $b_{i, j}$ (namely, $\widehat{b}_{i, j}$ ), is $n_{d}$ for all $k \in \mathbb{Z}_{0}^{+}$.

The discretized plant model (2.12) can be written as

$$
y(k)=\theta_{a}^{T} \varphi_{y}(k-1)+\sum_{i=1}^{n_{d}} \theta_{b, i}^{T} \bar{u}(k-i)+\Omega(k)=\theta^{T} \varphi(k-1)+\Omega(k),
$$


where

$$
\begin{aligned}
& \theta=\left[\begin{array}{lllll}
\theta_{a}^{T} & \theta_{b, 1}^{T} & \theta_{b, 2}^{T} & \cdots & \theta_{b, n_{d}}^{T}
\end{array}\right]^{T}, \quad \theta_{a}=\left[\begin{array}{llll}
-a_{1} & -a_{2} & \cdots & -a_{n_{0}}
\end{array}\right]^{T}, \\
& \theta_{b, i}=\left[\begin{array}{llll}
b_{i, 1} & b_{i, 2} & \cdots & b_{i, N}
\end{array}\right]^{T}, \\
& \varphi(k-1)=\left[\begin{array}{lllll}
\varphi_{y}^{T}(k-1) & \bar{u}^{T}(k-1) & \bar{u}^{T}(k-2) & \cdots & \bar{u}^{T}\left(k-n_{d}\right)
\end{array}\right]^{T}, \\
& \varphi_{y}(k-1)=\left[\begin{array}{llll}
y(k-1) & y(k-2) & \cdots & y\left(k-n_{0}\right)
\end{array}\right]^{T}, \\
& \bar{u}(k-i)=\left[\begin{array}{llll}
\alpha_{1} u(k-i) & \alpha_{2} u(k-i) & \cdots & \alpha_{N} u(k-i)
\end{array}\right]^{T}
\end{aligned}
$$

for $i \in\left\{1,2, \ldots, n_{d}\right\}$ and $j \in\{1, \ldots, N\}$. In the following, the case $N=n_{d}$ is considered.

\section{Adaptive Control Design}

The control objective is the adaptive stabilization of the continuous-time plant while matching, with a bounded tracking-error, a stable discrete-time free-design reference model $G_{m}(z)=B_{m}(z) / A_{m}(z)$ at the sampling instants. The perfect tracking is not achievable due to the presence of unknown unmodeled dynamics. A self-tuning regulator scheme is used to meet the control objective $[2,15]$. The control law structure is firstly presented for the nonadaptive case, that is, when the plant to be controlled is known. Then, an extension to the adaptive case is developed, which is the main interest of the paper. In such a case, a recursive algorithm of least-squares type with an adaptation dead-zone is used to obtain an estimation of the unknown parameters included in the vector $\theta$ of (2.17) at each sampling instant. Then, the multirate gains are updated in order to guarantee the inverse stability of the transferlike function associated to the discretized plant estimated model. Such a model is used to parameterize the adaptive controller.

\subsection{Known Plant}

The proposed control law is obtained from

$$
R(z) u(k)=T(z) c(k)-S(z) y(k)
$$

for all $k \in \mathbb{Z}_{0}^{+}$where $\{c(k)\}$ is the reference input sequence. The reconstruction of the plant input $u(t)$ is made by using (2.4), with the control sequence $\{u(k)\}$ obtained from (3.1) and the multirate gains $\alpha_{j}$, for all $j \in\{1, \ldots, N\}$, obtained from (2.15) with an appropriate choice of $v$ to guarantee the inverse stability of the discretized plant nominal model; that is, such gains fix the numerator $B_{d}(z)$ of $(2.14)$ to a prefixed one $B^{\prime}(z)$, whose coefficients are the components of $v$, with the roots within the stability domain. An important mathematical issue in the current context is that the proposed method allows the stabilization of the numerator polynomial of the discretized transfer function by an appropriate choice of the multirate gains even if its continuoustime counterpart is unstable or critically stable. 
The discrete-time transfer function of the closed-loop system obtained from the application of the control law (3.1) to the discretized plant model of transfer function (2.14) is given by

$$
\frac{Y(z)}{C(z)}=\frac{B^{\prime}(z) T(z)}{A(z) R(z)+B^{\prime}(z) S(z)}=\frac{T(z)}{A(z)+S(z)}
$$

if $R(z)=B^{\prime}(z)$ is chosen. In this way, the polynomial $B^{\prime}(z)$ is cancelled in the closed-loop system so that it does not generate discrete plant zeros. It should be stable (i.e., with zeros in $|z|<1)$ to cancel it if the usual methods without multirate techniques are used, [12, 14, 22]. Otherwise, it cannot be cancelled by the controller and it has then to be transmitted as a factor of the numerator of the closed-loop discrete transfer function (3.2). If it is transmitted, then the reference model is not of complete free choice since it has to contain this polynomial factor. The method proposed in this manuscript allows the stabilization of the discretized plant zeros. As a result, the reference model transfer function is always freely chosen with no zero transmission constraints by using the multirate technique with appropriate choice of the multirate gains. The control polynomials $T(z)$ and $S(z)$ to meet the model-matching objective are obtained from

$$
T(z)=B_{m}(z) A_{s}(z) \quad A(z)+S(z)=A_{m}(z) A_{s}(z)
$$

with the following degree constraints, required for controller realizability, in the controller synthesis:

$$
\begin{gathered}
\operatorname{Deg}\left[A_{m}(z)\right]=\operatorname{Deg}[A(z)]-\operatorname{Deg}\left[A_{s}(z)\right], \\
\operatorname{Deg}[S(z)]=\operatorname{Deg}[A(z)]-1=n_{d}-1=N-1, \\
\operatorname{Deg}[T(z)]=\operatorname{Deg}\left[B_{m}(z)\right]+\operatorname{Deg}\left[A_{s}(z)\right] \leq N-1,
\end{gathered}
$$

where $A_{s}(z)$ is a stable monic polynomial of zero-pole cancellations of the closed-loop system. In this way, the nominal closed-loop system matches the reference model at the sampling instants, but not the true closed-loop system due to the presence of unmodeled dynamics. However, the tracking-error is guaranteed to be bounded at all sampling times subject to Assumption 1(ii).

\subsection{Unknown Plant}

If the continuous-time plant parameters are unknown, then the vector $\theta$ in (2.17), which is composed of the discretized plant model parameters, is also unknown. However, all of the above control design in Section 3.1 remains valid if such a parameter vector is updated by an estimation algorithm. Such an algorithm provides an adaptation of each parameter $b_{i, j}$, namely, $\widehat{b}_{i, j}(k)$, for $i, j \in\{1, \ldots, N\}$ and all $k \in \mathbb{Z}_{0}^{+}$. Then, the multirate gains $\alpha_{j}$, now their estimates being $\widehat{\alpha}_{j}(k)$, are calculated from an equation similar to (2.15) by replacing the matrix $M$ by its estimated $\widehat{M}(k)$. In this way, the numerator of the corresponding discretized plant estimated model is fixed to $B^{\prime}(z)$. Note that such a numerator is time 


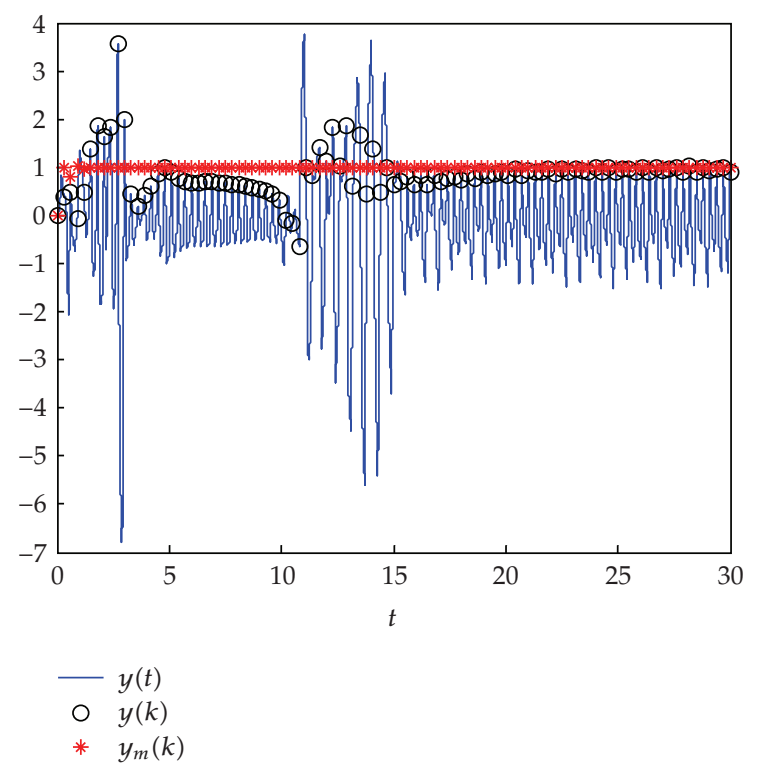

Figure 1: Continuous-time dynamic system and reference model measured signals.

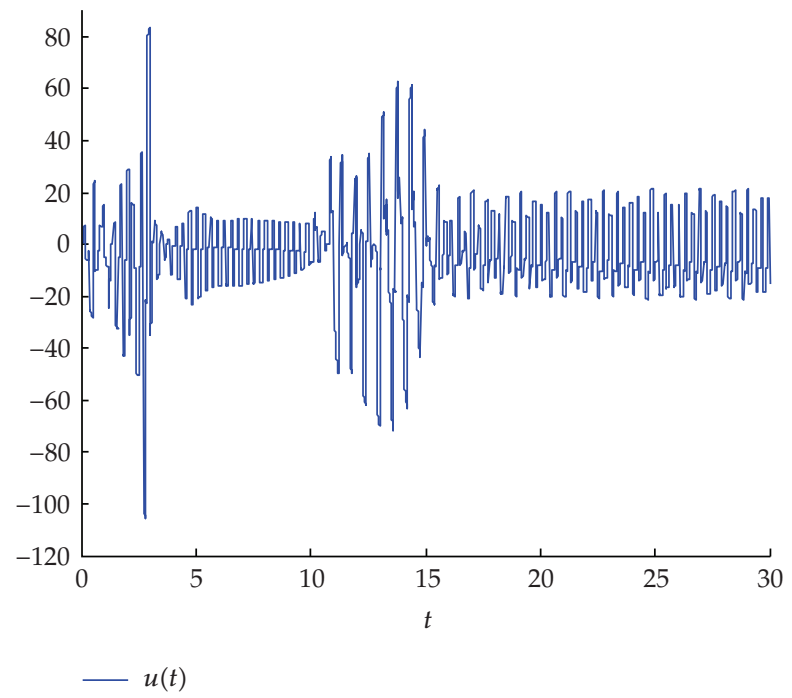

Figure 2: Continuous-time dynamic system control signal.

invariant although the estimates $\widehat{\theta}_{b, i}(k)$, for $i \in\{1, \ldots, N\}$, are time varying. Furthermore, the controller parameterization can be obtained from $R(z)=B^{\prime}(z)$ and equations similar to (3.3) by replacing the discretized plant polynomial $A(z)$ by its corresponding estimated one at the current sampling instant, namely, $\widehat{A}(z, k)$ [2]. In this context, the polynomials $T(z)$ and $R(z)$ have to be calculated once for all since $B_{m}(z), A_{s}(z)$ and $B^{\prime}(z)$, are time invariant. On the contrary, $S(z)$, now being $\widehat{S}(z, k)$, is updated at each running sampling instant since the polynomial $\widehat{A}(z, k)$ is time varying. 


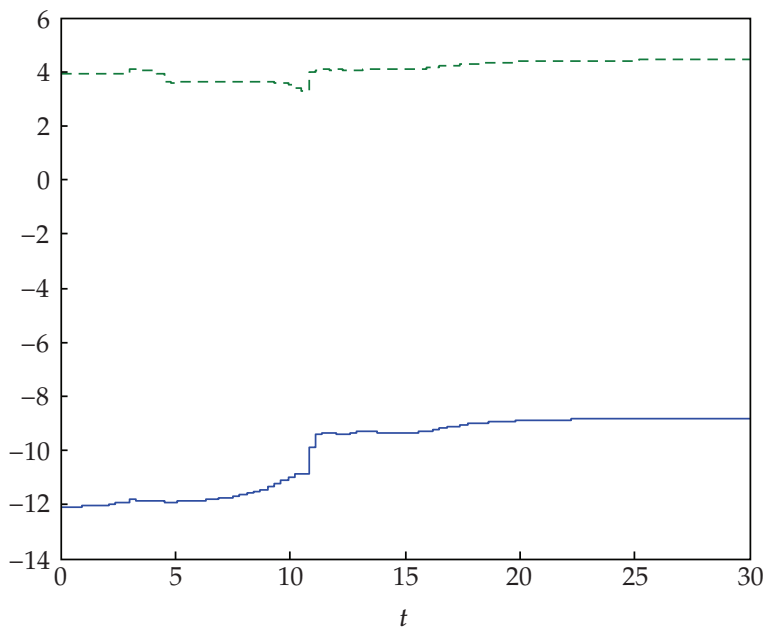

$-a_{1}$

Figure 3: Estimates of the parameters $a_{1}$ and $a_{2}$.

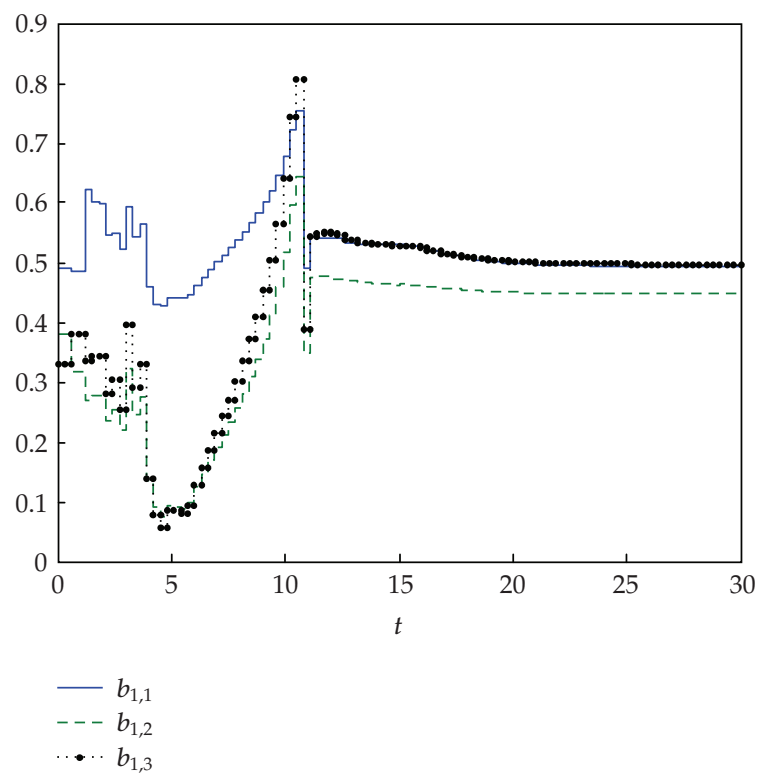

Figure 4: Estimates of the parameters $b_{1,1}, b_{1,2}$ and $b_{1,3}$. 


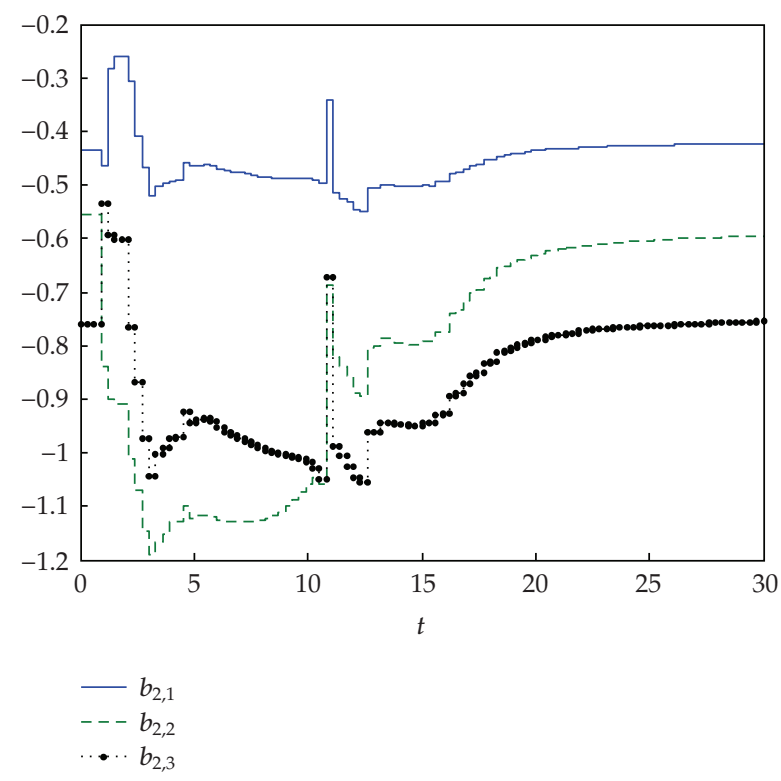

Figure 5: Estimates of the parameters $b_{2,1}, b_{2,2}$ and $b_{2,3}$.

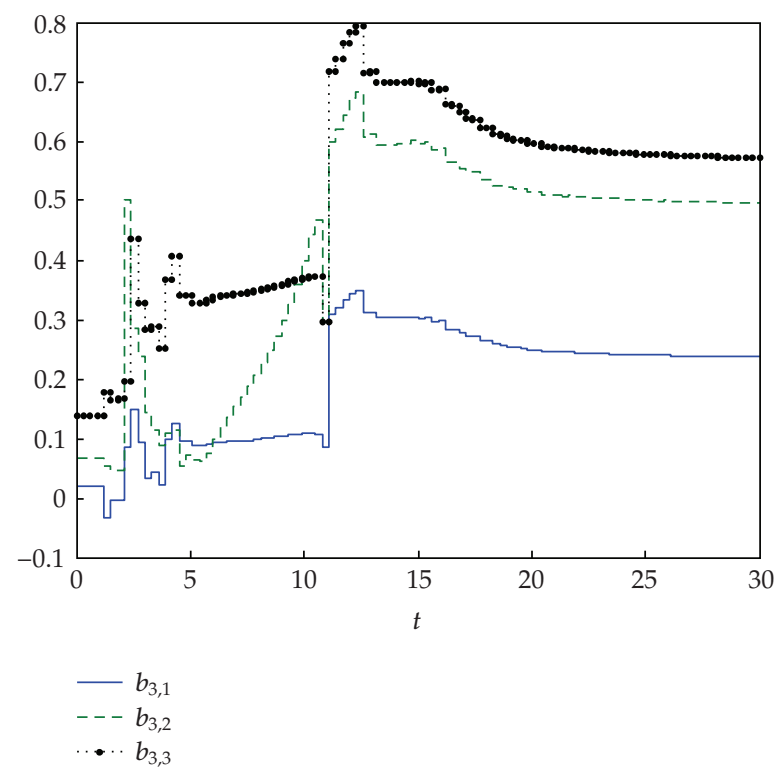

Figure 6: Estimates of the parameters $b_{3,1}, b_{3,2}$ and $b_{3,3}$. 


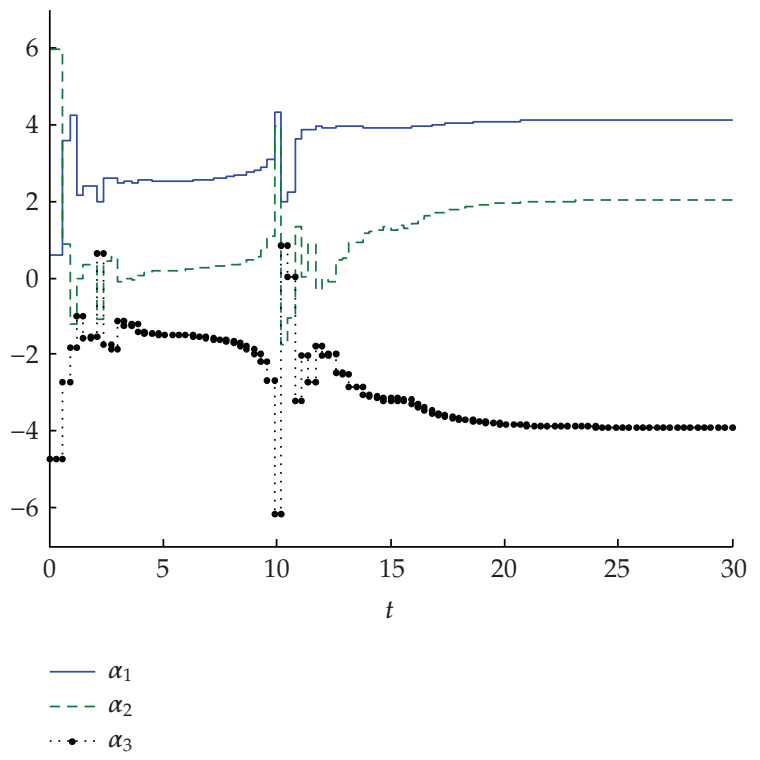

Figure 7: Multirate gains evolution.

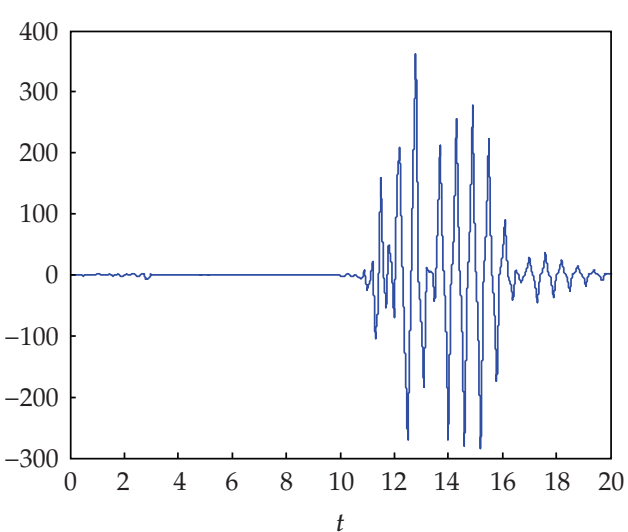

$-y(t)$

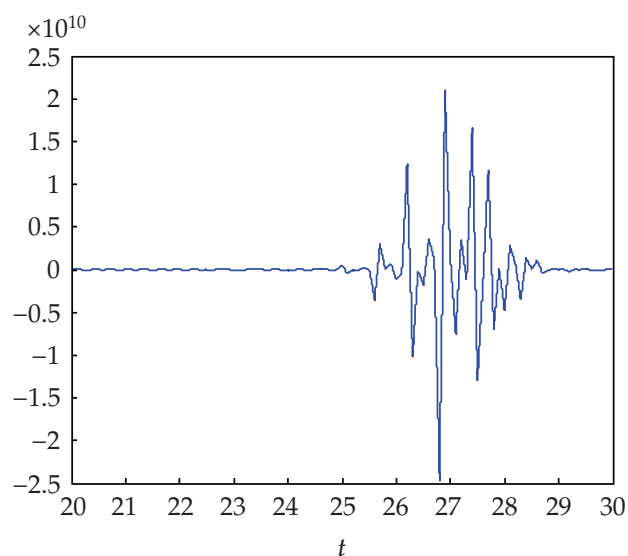

$-y(t)$

(a)

(b)

Figure 8: Continuous-time dynamic system measured signal without dead-zone in the parameters estimation algorithm. 
The time-varying multirate gains $\widehat{\alpha}_{j}(k)$ are used to calculate the plant input within the inter sample period via (2.4). Then, a time-varying input-output relation for the discretized plant is derived by following similar steps to those in Section 2.1. In this sense, one obtains

$$
\begin{aligned}
& y(k-\ell) \\
& =C_{0}\left\{F^{n_{0}-\ell} x_{0}\left(k-n_{0}\right)+\widehat{H}_{1}(k-\ell-1) u(k-\ell-1)+\sum_{i=\ell+1}^{n_{0}-1} F^{i-\ell-1}\left(F \widehat{H}_{1}(k-i-1)+\widehat{H}_{2}(k-i)\right)\right. \\
& \left.\times u(k-i-1)+F^{n_{0}-\ell-1} \widehat{H}_{2}\left(k-n_{0}\right) u\left(k-n_{0}-1\right)\right\}+\eta(k-\ell)
\end{aligned}
$$

for $\ell \in\left\{0,1, \ldots, n_{0}-1\right\}$, where $\widehat{H}_{1}(i)$ and $\widehat{H}_{2}(i)$ are obtained from equations similar to those in (2.6) by replacing $\alpha_{j}$ by $\widehat{\alpha}_{j}(i)$, for $i \in\left\{k-1, \ldots, k-n_{0}\right\}$ and $j \in\{1, \ldots, N\}$. Then

$$
Y_{v}(k-1)=V x_{0}\left(k-n_{0}\right)+\Pi_{v}(k-1)+\widehat{\Phi}\left(k-2, \ldots, k-n_{0}\right) U_{v}(k-2)
$$

where $Y_{v}(k-1), V, \Pi_{v}(k-1)$, and $U_{v}(k-2)$ are defined in (2.11) while

$$
\widehat{\Phi}\left(k-2, \ldots, k-n_{0}\right)=\left[\begin{array}{ccccc}
0 & 0 & \cdots & 0 & 0 \\
\widehat{\Phi}_{2,1} & \widehat{\Phi}_{2,2} & \cdots & 0 & 0 \\
\widehat{\Phi}_{3,1} & \widehat{\Phi}_{3,2} & \cdots & 0 & 0 \\
\vdots & \vdots & \ddots & \vdots & \vdots \\
\widehat{\Phi}_{n_{0}-1,1} & \widehat{\Phi}_{n_{0}-1,2} & \cdots & \widehat{\Phi}_{n_{0}-1, n_{0}-1} & 0 \\
\widehat{\Phi}_{n_{0}, 1} & \widehat{\Phi}_{n_{0}, 2} & \cdots & \widehat{\Phi}_{n_{0}, n_{0}-1} & \widehat{\Phi}_{n_{0}, n_{0}}
\end{array}\right]
$$

with $\widehat{\Phi}_{i, j}=C_{0} F^{i-j-1}\left(F \widehat{H}_{1}\left(k-n_{0}+j-2\right)+\widehat{H}_{2}\left(k-n_{0}+j-1\right)\right), \widehat{\Phi}_{i, i}=C_{0} \widehat{H}_{1}\left(k-n_{0}+i-2\right)$, and $\widehat{\Phi}_{i, 1}=C_{0} F^{i-2} \widehat{H}_{2}\left(k-n_{0}\right)$ for $i, j \in\left\{2,3, \ldots, n_{0}\right\}$ and $i>j$. By substituting in (3.5) the expression for $x_{0}\left(k-n_{0}\right)$, obtained from (3.6), for $\ell=0$ it follows that

$$
\begin{aligned}
y(k)= & -\sum_{i=1}^{n_{0}} a_{i} y(k-i)+C_{0} \widehat{H}_{1}(k-1) u(k-1) \\
& +\sum_{i=1}^{n_{0}-1}\left\{C_{0} F^{i-1}\left(F \widehat{H}_{1}(k-i-1)+\widehat{H}_{2}(k-i)\right)-\left[C_{0} F^{n_{0}} V^{-1} \widehat{\Phi}\left(k-2, \ldots, k-n_{0}\right)\right]_{n_{0}-i+1}\right\} \\
& \times u(k-i-1)+\left(C_{0} F^{n_{0}-1} \widehat{H}_{2}\left(k-n_{0}\right)-\left[C_{0} F^{n_{0}} V^{-1} \widehat{\Phi}\left(k-2, \ldots, k-n_{0}\right)\right]_{1}\right) u\left(k-n_{0}-1\right) \\
& +\sum_{i=1}^{n_{0}} a_{i} \eta(k-i)+\eta(k),
\end{aligned}
$$


or

$$
\begin{aligned}
y(k)= & -\sum_{i=1}^{n_{0}} a_{i} y(k-i)+C_{0} \widehat{H}_{1}(k-1) u(k-1) \\
& +\sum_{i=1}^{n_{0}-1}\left\{C_{0} F^{i-1}\left(F \widehat{H}_{1}(k-1)+\widehat{H}_{2}(k-1)\right)-\left[C_{0} F^{n_{0}} V^{-1} \widehat{\Phi}(k-1)\right]_{n_{0}-i+1}\right\} u(k-i-1) \\
& +\left(C_{0} F^{n_{0}-1} \widehat{H}_{2}(k-1)-\left[C_{0} F^{n_{0}} V^{-1} \widehat{\Phi}(k-1)\right]_{1}\right) u\left(k-n_{0}-1\right)+\sum_{i=1}^{n_{0}} a_{i} \eta(k-i) \\
& +\eta(k)+\Lambda_{\alpha}(k-1),
\end{aligned}
$$

where

$$
\begin{aligned}
& \Lambda_{\alpha}(k-1) \\
& =\sum_{i=1}^{n_{0}-1}\left\{C_{0} F^{i-1}\left(F \Delta \widehat{H}_{1}(k-i-1)+\Delta \widehat{H}_{2}(k-i)\right)-\left[C_{0} F^{n_{0}} V^{-1} \Delta \widehat{\Phi}\right]_{n_{0}-i+1}\right\} u(k-i-1) \\
& \quad+\left(C_{0} F^{n_{0}-1} \Delta \widehat{H}_{2}\left(k-n_{0}\right)-\left[C_{0} F^{n_{0}} V^{-1} \Delta \widehat{\Phi}\right]_{1}\right) u\left(k-n_{0}-1\right)
\end{aligned}
$$

with $\Delta \widehat{H}_{\ell}(k-i-1)=\widehat{H}_{\ell}(k-i-1)-\widehat{H}_{\ell}(k-1)$, for $\ell=1,2$, and $\Delta \widehat{\Phi}=\widehat{\Phi}\left(k-2, \ldots, k-n_{0}\right)-\widehat{\Phi}(k-1)$. Note that $\Omega(k)=\sum_{i=1}^{n_{0}} a_{i} \eta(k-i)+\eta(k)$ arises from the unmodeled dynamics of the continuoustime plant while $\Lambda_{\alpha}(k-1)$ comes from the fact that the multirate gains are time varying. Both terms constitute the unmodeled dynamics for the discretized plant model. On one hand, the latter tends to zero if the multirate gains converge to constant values. On the other hand, the former is such that

$$
|\Omega(k)| \leq \bar{\Omega}(k)=\mu_{1} \rho(k)+\mu_{2}
$$

for some known real constants $\mu_{1} \geq 0$ and $\mu_{2} \geq 0$, where

$$
\rho(k)=\operatorname{Sup}_{0 \leq k^{\prime} \leq k}\left\{\left|w^{T} x\left(k^{\prime}\right)\right| \sigma^{k-k^{\prime}}\right\}
$$

for all $k \in \mathbb{Z}_{0}^{+}$, some known constant vector $w$, and some known real constant $\sigma \in(0,1)$ with

$$
x(k)=\left[\begin{array}{llllll}
y(k-1) & \cdots & y\left(k-n_{0}\right) & u(k-1) & \cdots & u(k-N)
\end{array}\right]^{T}
$$


in view of Assumption 1(ii) [14]. Finally, one can express the discretized plant model as

$$
\begin{aligned}
y(k) & =-\sum_{i=1}^{n_{0}} a_{i} y(k-i)+\sum_{i=1}^{n_{d}} b_{i}(k-1) u(k-i)+\Omega(k)+\Lambda_{\alpha}(k-1) \\
& =-\sum_{i=1}^{n_{0}} a_{i} y(k-i)+\sum_{i=1}^{n_{d}} \sum_{j=1}^{N} b_{i, j} \widehat{\alpha}_{j}(k-1) u(k-i)+\Omega(k)+\Lambda_{\alpha}(k-1) \\
& =\theta^{T} \widehat{\varphi}(k-1)+\Omega(k)+\Lambda_{\alpha}(k-1),
\end{aligned}
$$

where $\widehat{\varphi}(k-1)$ is built like $\varphi(k-1)$ in (2.17) by replacing the components of $\bar{u}(k-i)$, namely, $\alpha_{j} u(k-i)$, by the corresponding $\widehat{\alpha}_{j}(k-1) u(k-i)$, for $i, j \in\{1, \ldots, N\}$.

The algorithm used to obtain an on-line adaptation $\hat{\theta}(k)$ of the unknown parameters vector $\theta$ is described below.

\subsubsection{Estimation Algorithm}

An "a priori" estimated parameters vector is obtained by using a recursive least-squares algorithm defined by

$$
\begin{aligned}
\widehat{\theta}^{0}(k) & =\widehat{\theta}^{0}(k-1)+\frac{s(k) P(k-1) \widehat{\varphi}_{n}(k-1) e_{n}^{0}(k)}{r(k)+s(k) \widehat{\varphi}_{n}^{T}(k-1) P(k-1) \widehat{\varphi}_{n}(k-1)}, \\
P(k) & =P(k-1)-\frac{s(k) P(k-1) \widehat{\varphi}_{n}(k-1) \widehat{\varphi}_{n}^{T}(k-1) P(k-1)}{r(k)+s(k) \hat{\varphi}_{n}^{T}(k-1) P(k-1) \hat{\varphi}_{n}(k-1)}
\end{aligned}
$$

for all $k \in \mathbb{Z}^{+}$, where $\widehat{\varphi}_{n}(k-1)=\widehat{\varphi}(k-1) /(1+\|\widehat{\varphi}(k-1)\|), P(k-1)$ is initialized such that $P(0)=P^{T}(0)>0\left(\right.$ denoting positive definiteness), $\gamma(k)>0, e_{n}^{0}(k)=e^{0}(k) /(1+\|\widehat{\varphi}(k-1)\|)$ with

$$
e^{0}(k)=-\widetilde{\theta}^{0 T}(k-1) \widehat{\varphi}(k-1)+\Omega(k)+\Lambda_{\alpha}(k-1)
$$

being the "a priori" estimation error as well as $\widetilde{\theta}^{0}(k-1)=\widehat{\theta}^{0}(k-1)-\theta$ the "a priori" parametrical error, and $s(k)$ is a relative adaptation dead-zone defined as

$$
s(k)= \begin{cases}0 & \text { if } e_{a n}^{0}(k) \leq \mu \bar{\eta}_{a n}(k), \\ \frac{f(k)}{e_{a n}^{0}(k)} & \text { otherwise }\end{cases}
$$

for some $\mu>1$, where $e_{a n}^{0}(k)=\left(\left(e_{n}^{0}(k)\right)^{2}+\widehat{\varphi}_{n}^{T}(k-1) P^{2}(k-1) \widehat{\varphi}_{n}(k-1)\right)^{1 / 2}$ is an augmented normalized error, $\bar{\eta}_{a n}(k)=\left(1+\gamma^{-1}(k) \widehat{\varphi}_{n}^{T}(k-1) P(k-1) \widehat{\varphi}_{n}(k-1)\right)^{1 / 2} \bar{\eta}_{T n}(k)$ with $\bar{\eta}_{T n}(k)=$ 
$\bar{\eta}_{T}(k) /(1+\|\varphi(k-1)\|)$ and $\bar{\eta}_{T}(k)$ being an upper bound for $\left|\eta_{T}(k)\right|=\left|\Omega(k)+\Lambda_{\alpha}(k-1)\right|$, and

$$
f(k)= \begin{cases}0 & \text { if } e_{a n}^{0}(k) \leq \mu \bar{\eta}_{a n}(k), \\ e_{a n}^{0}(k)-\mu \bar{\eta}_{a n}(k) & \text { otherwise. }\end{cases}
$$

This algorithm provides an estimation $\widehat{\theta}^{0}(k)$ of the parameters vector. Then, an "a posteriori" estimates vector is obtained as follows.

\section{Estimates Modification}

This algorithm consists of three main steps as follows.

Step 1. Build the matrix $\widehat{M}^{0}(k)=\left[\widehat{b}_{i, j}^{0}(k)\right] \in \mathbb{R}^{N \times N}$, for $i, j \in\{1,2, \ldots, N\}$, from the "a priori" estimates $\widehat{\theta}_{b, i}^{0}(k)$, included in $\widehat{\theta}^{0}(k)$, of the corresponding $\theta_{b, i}$ defined in (2.17).

Step 2. $\widehat{M}(k)=\widehat{M}^{0}(k)$ :

$$
\begin{array}{r}
\text { If }|\operatorname{Det}(\widehat{M}(k))| \geq \delta_{0} \text { then } \widehat{\theta}_{b, i}(k)=\widehat{\theta}_{b, i}^{0}(k) \\
\text { else while }|\operatorname{Det}(\widehat{M}(k))|<\delta_{0} \\
\widehat{M}(k)=\widehat{M}(k)+\delta I_{N}
\end{array}
$$

end;

for $i=1$ to $N$

$$
\widehat{\theta}_{b, i}(k)=\widehat{M}_{i}(k)
$$

end,

end.

Step 3. $\widehat{\theta}(k)=\left[\begin{array}{lllll}\hat{\theta}_{a}^{0 T}(k) & \widehat{\theta}_{b, 1}^{T}(k) & \widehat{\theta}_{b, 2}^{T}(k) & \cdots & \widehat{\theta}_{b, N}^{T}(k)\end{array}\right]^{T}$,

for some positive real constants $\delta \ll 1$ and $\delta_{0} \ll 1$, and where $\widehat{M}_{i}(k)$ denotes the $i$-th row of $\widehat{M}(k)$ and $I_{N}$ the identity matrix of dimension $N \times N$.

Once the estimated parameters are updated, the multirate gains vector $\hat{g}(k)=$ $\left[\begin{array}{lll}\widehat{\alpha}_{1}(k) & \cdots & \widehat{\alpha}_{N}(k)\end{array}\right]^{T}$ is calculated from an equation similar to $(2.15)$ by replacing the matrix $M$ by $\widehat{M}(k)=\left[\widehat{b}_{i, j}(k)\right] \in \mathbb{R}^{N \times N}$.

Remark 3.1. (i) The proposed estimates modification process avoids that the time-varying matrix $\widehat{M}(k)$ be close to a singular one. In this way, a bounded vector of multirate gains is obtained at all sampling instants.

(ii) Note that the estimate $\widehat{\theta}_{a}^{0}(k)$ corresponding to the parameters of $\theta_{a}$ is not affected by the modification algorithm. In fact, such a modification only affects the entries in the main diagonal of $\widehat{M}^{0}(k)$. Also, note that the instruction while of the second step is executed a finite number of times since there exists a finite integer number $\ell$ such that $|\operatorname{Det}[\widehat{M}(k)]|=$ $\left|\operatorname{Det}\left[\widehat{M}^{0}(k)+\ell \delta I_{N}\right]\right|=\left|(\ell \delta)^{N}+f_{0}\left(\delta, \widehat{\theta}_{b, i}^{0}(k)\right)\right| \geq \delta_{0}$ for $i \in\{1, \ldots, N\}$ and some function $f_{0}(\cdot, \cdot)$. 
(iii) From (3.10) to (3.13) and the construction of $\widehat{\varphi}(k-1)$, there exist some real constants $v_{i} \geq 0$, for $i \in\{1,2,3,4\}$ such that the sequences $\left\{\bar{\eta}_{1}(k)\right\}$, with $\bar{\eta}_{1}(k)=v_{1} \| \widehat{\varphi}(k-$ 1) $\|+v_{2}$, and $\left\{\bar{\eta}_{2}(k)\right\}$, with $\bar{\eta}_{2}(k)=v_{3}\|x(k-1)\|+v_{4}$, are both upper bounds for $\left\{\left|\eta_{T}(k)\right|\right\}$.

The estimation algorithm together with such a multirate gains adaptation possesses the following properties.

Lemma 3.2 (main properties of the estimation algorithm). (i) $P(k)$ is uniformly bounded for all $k \in \mathbb{Z}_{0}^{+}$, and it asymptotically converges to a finite, at least semidefinite positive, limit as $k \rightarrow \infty$.

(ii) $\widehat{\theta}^{0}(k)$ and $\widehat{\theta}(k)$ are uniformly bounded for all samples and converge to finite limits.

(iii) $f(k)<\infty$ for all $k \in \mathbb{Z}_{0}^{+}$and $\lim _{k \rightarrow \infty} f(k)=0$.

(iv) The vector $\widehat{g}(k)$ is bounded for all samples and converges to a finite limit.

The proof is made in Appendix A.

\section{Stability Analysis}

The plant discretized model can be written as follows:

$$
\begin{aligned}
y(k) & =\widehat{y}(k)+e(k)=\widehat{\theta}^{T}(k-1) \widehat{\varphi}(k-1)+e(k) \\
& =-\sum_{i=1}^{n_{0}} \widehat{a}_{i}(k-1) y(k-i)+\sum_{i=1}^{N} \sum_{j=1}^{N} \widehat{b}_{i, j}(k-1) \widehat{\alpha}_{j}(k-1) u(k-i)+e(k) \\
& =-\sum_{i=1}^{n_{0}} \widehat{a}_{i}(k-1) y(k-i)+\sum_{i=1}^{N} b_{i}^{\prime} u(k-i)+e(k)
\end{aligned}
$$

and the adaptive control law (3.1), replacing $S(z)$ by $\widehat{S}(k, z)$, as

$$
\begin{aligned}
u(k)=\frac{1}{b_{1}^{\prime}}\{ & \sum_{i=1}^{N-1}\left(\widehat{s}_{1}(k-1) \widehat{a}_{i}(k-1)-\widehat{s}_{i+1}(k-1)\right) y(k-i) \\
& +\sigma(\beta) \widehat{s}_{1}(k-1) \widehat{a}_{N}(k-1) y(k-N)-\sum_{i=1}^{N-1}\left(\widehat{s}_{1}(k-1) b_{i}^{\prime}+b_{i+1}^{\prime}\right) u(k-i) \\
& \left.-\widehat{s}_{1}(k-1) b_{N}^{\prime} u(k-N)+\sum_{i=1}^{N} t_{i} c(k-i+1)-\widehat{s}_{1}(k-1) e(k)\right\}
\end{aligned}
$$

where $\widehat{S}(z, k-1)=\sum_{i=1}^{N} \widehat{s}_{i}(k-1) z^{N-i}, T(z)=\sum_{i=1}^{N} t_{i} z^{N-i}, R(z)=B^{\prime}(z)=\sum_{i=1}^{N} b_{i}^{\prime} z^{N-i}$, and the binary-valued function

$$
\sigma(\beta)= \begin{cases}1 & \text { if } \beta=0 \\ 0 & \text { otherwise }\end{cases}
$$


have been used. By combining (4.1) and (4.2), the discrete-time closed-loop system can be written as

$$
x(k)=\Lambda(k-1) x(k-1)+\Psi_{1}(k-1) e(k)+\frac{1}{b_{1}^{\prime}} \Psi_{2} \sum_{i=1}^{N} t_{i} c(k-i+1),
$$

where

$$
\begin{aligned}
& x(k)=\left[\begin{array}{llllllll}
y(k) & y(k-1) & \cdots & y\left(k-n_{0}+1\right) & u(k) & u(k-1) & \cdots & u(k-N+1)
\end{array}\right]^{T}, \\
& \Psi_{1}(k-1)=\left[\begin{array}{llllllll}
1 & 0 & \cdots & 0 & \underbrace{-\widehat{s}_{1}(k-1) / b_{1}^{\prime}}_{n_{0}+1} & 0 & \cdots & 0
\end{array}\right]^{T} \in \mathbb{R}^{\left(n_{0}+N\right) \times 1}, \\
& \Psi_{2}=\left[\begin{array}{llllllll}
0 & 0 & \cdots & 0 & \underbrace{1}_{n_{0}+1} & 0 & \cdots & 0
\end{array}\right]^{T} \in \mathbb{R}^{\left(n_{0}+N\right) \times 1},
\end{aligned}
$$

$\Lambda(k-1)$

$$
=\left[\begin{array}{ccccc|ccccc}
-\widehat{a}_{1}(k-1) & -\widehat{a}_{2}(k-1) & \cdots & -\widehat{a}_{n_{0}-1}(k-1) & -\widehat{a}_{n_{0}}(k-1) & b_{1}^{\prime} & b_{2}^{\prime} & \cdots & b_{N-1}^{\prime} & b_{N}^{\prime} \\
1 & 0 & \cdots & 0 & 0 & 0 & 0 & \cdots & 0 & 0 \\
0 & 1 & \cdots & 0 & 0 & 0 & 0 & \cdots & 0 & 0 \\
\vdots & \vdots & \ddots & \vdots & \vdots & \vdots & \vdots & \ddots & \vdots & \vdots \\
0 & 0 & \cdots & 1 & 0 & 0 & 0 & \cdots & 0 & 0 \\
\hline \widehat{f}_{1}(k-1) & \widehat{f}_{2}(k-1) & \cdots & \widehat{f}_{n_{0}-1}(k-1) & \widehat{f}_{n_{0}}(k-1) & -\widehat{h}_{1}(k-1) & -\widehat{h}_{2}(k-1) & \cdots & -\widehat{h}_{N-1}(k-1) & -\widehat{h}_{N}(k-1) \\
0 & 0 & \cdots & 0 & 0 & 1 & 0 & \cdots & 0 & 0 \\
0 & 0 & \cdots & 0 & 0 & 0 & 1 & \cdots & 0 & 0 \\
\vdots & \vdots & \ddots & \vdots & \vdots & \vdots & \vdots & \ddots & \vdots & \vdots \\
0 & 0 & \cdots & 0 & 0 & 0 & 0 & \cdots & 1 & 0
\end{array}\right]
$$

with $\widehat{f}_{n_{0}}(k-1)=\left(1 / b_{1}^{\prime}\right)\left[\widehat{s}_{1}(k-1) \widehat{a}_{n_{0}}(k-1)-(1-\sigma(\beta)) \widehat{s}_{n_{0}+1}(k-1)\right], \widehat{f}_{i}(k-1)=\left(1 / b_{1}^{\prime}\right)\left[\widehat{s}_{1}(k-\right.$ 1) $\left.\widehat{a}_{i}(k-1)-\widehat{s}_{i+1}(k-1)\right]$, for $i \in\left\{1,2, \ldots, n_{0}-1\right\}, \widehat{h}_{N}(k-1)=\left(b_{N}^{\prime} / b_{1}^{\prime}\right) \widehat{s}_{1}(k-1)$, and $\widehat{h}_{i}(k-1)=$ $\left(1 / b_{1}^{\prime}\right)\left[\widehat{s}_{1}(k-1) b_{i}^{\prime}+b_{i+1}^{\prime}\right]$ for $i \in\{1,2, \ldots, N-1\}$. Note that $\widehat{a}_{i}(k-1)$ are uniformly bounded from Lemma 3.2. Also, $\widehat{s}_{i}(k-1)$ are uniformly bounded from the resolution of an equation similar to (3.3) by replacing the polynomials $A(z)$ and $S(z)$ by $\widehat{A}(k-1, z)$ and $\widehat{S}(k-1, z)$, respectively. The following theorem, whose proof is made in Appendix $B$, establishes the main stability result of the adaptive control system.

Theorem 4.1 (main stability result). (i) The adaptive control law stabilizes the plant model (3.14) in the sense that $\{u(k)\}$ and $\{y(k)\}$ are bounded for all finite initial states and any bounded reference input sequence $\{c(k)\}$ subject to Assumption 1.

(ii) The control and measured signals of the continuous-time dynamic system, $u(t)$ and $y(t)$, are bounded for all $t$. 


\section{Simulations}

A continuous-time dynamic system defined by the matrices

$$
A=\left[\begin{array}{cc|cc}
0 & 17.5 & 0 & 0 \\
1 & 1.5 & 0 & 0 \\
\hline 0 & 0 & -10 & 0 \\
0 & 1 & 0 & -15
\end{array}\right], \quad B=\left[\begin{array}{c}
-1 \\
1 \\
0.05 \\
0
\end{array}\right], \quad C=\left[\begin{array}{lll}
0 & 1 \mid & 0.05
\end{array}\right]
$$

in the state-space and by the transfer function

$$
G(s)=\frac{s-1}{(s-5)(s+3.5)}\left(1+\frac{0.05}{s+15}\right)+\frac{0.05}{s+10}
$$

is considered. This plant is supposed unknown and the adaptive control strategy described in the paper will be used to stabilize it. Such a strategy is based on a discretization process using a FROH with $\beta=0.3$ for a slow sampling time $T=0.3$ and a multirate device with $N=3$ to place the zeros of the estimated discretized plant model within the stability domain. The time-varying discrete transfer-like function corresponding to such an estimated model is $\widehat{G}_{d}(z, k)=\left(\widehat{B}_{d}(z, k) / \widehat{A}_{d}(z, k)\right)=\left(\left(\widehat{b}_{1}(k) z^{2}+\widehat{b}_{2}(k) z+\widehat{b}_{3}(k)\right) / z\left(z^{2}+\widehat{a}_{1}(k) z+\widehat{a}_{2}(k)\right)\right)$ with $\widehat{b}_{i}(k)=\sum_{j=1}^{3} \widehat{b}_{i j}(k) \widehat{\alpha}_{j}(k)$, for all $i \in\{1,2,3\}$ and all $k \in \mathbb{Z}_{0}^{+}$. The estimates $\widehat{b}_{i j}(k)$ are provided by the estimation algorithm described in Section 3.2.1 with $\gamma(k)=0.01$ for all $k \in \mathbb{Z}_{0}^{+}$and initialized with $P(0)=55 \times I_{11}, \widehat{\theta}_{a}(0)=\left[\begin{array}{lll}-12.079 & 3.92\end{array}\right]^{T}, \widehat{\theta}_{b, 1}(0)=\left[\begin{array}{lll}0.492 & 0.379 & 0.33\end{array}\right]^{T}$, $\widehat{\theta}_{b, 2}(0)=\left[\begin{array}{lll}-0.435 & -0.556 & -0.759\end{array}\right]^{T}$, and $\widehat{\theta}_{b, 3}(0)=\left[\begin{array}{lll}0.019 & 0.066 & 0.139\end{array}\right]^{T}$. Also, $\bar{\eta}_{T}(k)=$ $v_{1}\|\hat{\varphi}(k-1)\|+v_{2}$, with $v_{1}=0.0055$ and $v_{2}=0.01$, is used as upper bound for $\left|\eta_{T}(k)\right|$ and $\mu=1.1$ for the dead-zone included in such an algorithm. The values $\delta=\delta_{0}=10^{-6}$ are taken in Step 2 of the estimates modification procedure. The gains $\widehat{\alpha}_{j}(k)$, for $j \in\{1,2,3\}$, are on-line updated in order to fix $\widehat{B}(z, k)$ to the polynomial $B^{\prime}(z)=z^{2}-0.25$. The control objective is to match the reference model given by $G_{m}(z)=\left(\left(z^{2}+0.1 z+0.083\right) /\left(z^{3}+\right.\right.$ $\left.\left.0.3 z^{2}-0.09 z-0.027\right)\right)$. Figures 1 and 2 display, respectively, the continuous-time dynamic system measured and control signals under a unitary step external input sequence $\{c(k)\}$. Note that both signals are bounded for all time; that is, the stabilization of the closed-loop system is reached. Furthermore, the plant output sequence $\{y(k)\}$ converges asymptotically to $\left\{y_{m}(k)\right\}$. Figures $3,4,5$, and 6 show the evolution of the estimates, included as components of $\widehat{\theta}(k)=\left[\begin{array}{llll}\widehat{\theta}_{a}^{T}(k) & \widehat{\theta}_{b, 1}^{T}(k) & \widehat{\theta}_{b, 2}^{T}(k) & \widehat{\theta}_{b, 3}^{T}(k)\end{array}\right]^{T}$, during the simulation while Figure 7 displays the evolution of the multirate gains $\widehat{g}(k)=\left[\begin{array}{lll}\widehat{\alpha}_{1}(k) & \widehat{\alpha}_{2}(k) & \widehat{\alpha}_{3}(k)\end{array}\right]^{T}$. Note that therefore the estimated parameters as the multirate gains converge to a set of constant values as $t$ tends to infinite. Finally, Figures 8(a) and 8(b) show the evolution of the continuous-time dynamic system measured signal, each one in a different time interval, if the same estimation algorithm without the dead-zone is used to stabilize the system. The behavior of the adaptive control system is improved with the inclusion of the relative adaptation dead-zone in this particular example as one can see by comparing the signal $y(t)$ in Figure 1 with those displayed in Figures $8(a)$ and $8(b)$. This conclusion cannot be generalized for all cases since one can search examples where the inclusion of the dead-zone does not improve the performance of the control system. However, the inclusion of the relative dead-zone guarantees the stabilization 
of the adaptive control system under the presence of unmodeled dynamics, which is what justifies its inclusion in the parameters estimation algorithm.

\section{Conclusions}

An adaptive control strategy for stabilizing linear time-invariant continuous-time dynamic systems, being possibly of inverse nonstable, and subject to the presence of unmodeled dynamics has been presented. The control design is based on the discrete-time model reference adaptive (MRAC) control method. Therefore, an inversely stable discretized model of the continuous-time dynamic system is required to achieve the stabilization objective while matching a freely chosen discrete-time reference model. Such a requirement is guaranteed by using a fractional-order hold $(\mathrm{FROH})$ combined with a multirate device with fast sampling input in the discretization process of the continuous-time system. In this context, an estimation algorithm is used to on-line update the multirate gains such that the zeros of the transfer-like function associated to the estimated model of the discretized system are within the open-unit complex circle at all sampling instants. The parameters of such an estimated model are used to on-line parameterize the adaptive control law. The estimation algorithm includes a relative adaptation dead-zone for dealing with the presence of unmodeled dynamics. The stability of the adaptive control system is proved under the assumption that the nominal model of the continuous-time dynamic system is observable, an upper-bound of its order is known, and the contribution of the unmodeled dynamics to the system output is sufficiently small. Finally, the performance of the adaptive control system is shown by means of simulation results. The stabilization of the system is manifested although the intersample behavior of the measured signal could be improved. A future potential research may be the use of the same control technique in a multiestimation scheme for improving such an intersample behavior.

\section{Appendices}

\section{A. Proof of Lemma 3.2}

(i) Equation (32b) and the matrix inversion lemma lead to $P^{-1}(k)=P^{-1}(k-1)+$ $\gamma^{-1}(k) s(k) \widehat{\varphi}_{n}(k-1) \widehat{\varphi}_{n}^{T}(k-1)>0$ for all $k \in \mathbb{Z}^{+}$provided that $P(0)=P^{T}(0)>0$. Then, $\{P(k)\}$ is a nonnegative and monotonic nonincreasing matrix sequence. Thus, $0 \leq P(k) \leq P(0)$ and $P(k)$ asymptotically converges to a finite limit as $k \rightarrow \infty$.

(ii) By considering the nonnegative sequence $V(k)=\tilde{\theta}^{0 T}(k) P^{-1}(k) \tilde{\theta}^{0}(k)+\operatorname{Tr}\{P(k)\}$ and using the matrix inversion lemma in $(32 b)$, it follows that

$$
\begin{aligned}
V(k)-V(k-1) & =-\frac{s(k)\left(\left(e_{a n}^{0}(k)\right)^{2}-\left(\eta_{a n}(k)\right)^{2}\right)}{\gamma(k)+s(k) \widehat{\varphi}_{n}^{T}(k-1) P(k-1) \hat{\varphi}_{n}(k-1)} \\
& \leq-\frac{\left(\left(\mu^{2}-1\right) / \mu^{2}\right) s(k)\left(e_{a n}^{0}(k)\right)^{2}}{r(k)+s(k) \hat{\varphi}_{n}^{T}(k-1) P(k-1) \widehat{\varphi}_{n}(k-1)} \\
& \leq-\frac{\left(\left(\mu^{2}-1\right) / \mu^{2}\right)(f(k))^{2}}{r(k)+s(k) \widehat{\varphi}_{n}^{T}(k-1) P(k-1) \hat{\varphi}_{n}(k-1)} \leq 0,
\end{aligned}
$$


where (32a) and the definition of the "a priori" estimation error have been used. Then, $V(k) \leq$ $V(0)<\infty$ and $\left\|\widetilde{\theta}^{0}(k)\right\| \leq\left(\lambda_{\max }\{P(0)\} / \lambda_{\min }\{P(0)\}\right)\left\|\tilde{\theta}^{0}(0)\right\|+\lambda_{\max }\{P(0)\} \operatorname{Tr}\{P(0)\}<\infty$ where $\lambda_{\max }(M)$ and $\lambda_{\min }(M)$ denote the maximum and the minimum eigenvalues of the matrix $M$, respectively. It implies that $\widetilde{\theta}^{0}(k)$ and then also $\widehat{\theta}^{0}(k)$ are uniformly bounded. Then, $\hat{\theta}(k)$ is also bounded since the modification algorithm guarantees the boundedness of $\widehat{M}(k)$ provided that $\widehat{\theta}^{0}(k)$ is bounded. Moreover, $V(k)$ asymptotically converges to a finite limit as $k \rightarrow \infty$ from its definition and the fact that such a sequence is nonnegative and monotonic nonincreasing. Then, $\widetilde{\theta}^{0}(k)$, and also $\widehat{\theta}^{0}(k)$, converges to a finite limit as $k \rightarrow \infty$ since $P(k)$ also converges as it has been proved in (i). Then, $\widehat{M}(k)$ and $\widehat{\theta}(k)$ also converge to finite limits as $k \rightarrow \infty$.

(iii) From (A.1), it follows that

$$
\frac{\mu^{2}-1}{\mu^{2}} \sum_{i=1}^{k} \frac{(f(i))^{2}}{\gamma(i)+s(i) \hat{\varphi}_{n}^{T}(i-1) P(i-1) \widehat{\varphi}_{n}(i-1)} \leq V(0)-V(k) \leq V(0)<\infty
$$

for all $k \in \mathbb{Z}^{+}$. Then $f(k)<\infty$ for all $k \in \mathbb{Z}^{+}$and also $\lim _{k \rightarrow \infty} f(k)=0$.

(iv) The boundedness and convergence of the estimation model parameters vector together with the nonsingularity of matrix $\widehat{M}(k)$ (guaranteed by the modification algorithm) imply the boundedness and convergence of the vector $\widehat{g}(k)$ obtained by resolution of $(2.15)$ replacing $M$ by $\widehat{M}(k)$.

\section{B. Proof of Theorem 4.1}

(i) $\Lambda(k-1)$ is bounded since the estimated plant parameters $\widehat{a}_{i}(k-1)$, for $i \in\left\{1, \ldots, n_{0}\right\}$, and the controller parameters $\widehat{s}_{j}(k-1)$, for $j \in\{1, \ldots, N\}$, are bounded thanks to $\widehat{\theta}(k-1)$ and $\hat{g}(k-1)$ are bounded for all $k \in \mathbb{Z}^{+}$, see Lemma 3.2. The eigenvalues of $\Lambda(k-1)$ are in $|z|<1$ since they are the roots of $A_{m}(z), A_{s}(z)$ and $B^{\prime}(z)$, which are stable. Furthermore,

$$
\sum_{k^{\prime}=k_{0}+1}^{k}\left\|\Lambda\left(k^{\prime}\right)-\Lambda\left(k^{\prime}-1\right)\right\|^{2} \leq \gamma_{0}+\gamma_{1}\left(k-k_{0}\right)
$$

for all integers $k>k_{0} \geq 0$ and some positive real constants $\gamma_{0}$ and $\gamma_{1}$, with $\gamma_{1}$ being sufficiently small by using slow enough estimation rates via a suitable $P(0)$ in (32b). Thus, the unforced time-varying system $x(k)=\Lambda(k-1) x(k-1)$ is exponentially stable and its transition matrix $\phi\left(k, k^{\prime}\right)=\prod_{j=k^{\prime}}^{k-1} \Lambda(j)$ satisfies $\left\|\phi\left(k, k^{\prime}\right)\right\| \leq \rho_{1} \sigma_{0}^{k-k^{\prime}}$ for all integer $k \geq k^{\prime}$ where $\sigma_{0} \in(0,1)$ is an upper bound for the absolute value of the closed-loop stability abscissa and $\rho_{1}$ is a nondependent constant [2]. Note that $\sigma_{0}$ depends on the freely chosen reference model. From (4.4),

$$
x(k)=\phi\left(k, k_{0}\right) x\left(k_{0}\right)+\sum_{k^{\prime}=k_{0}}^{k} \phi\left(k, k^{\prime}\right)\left(\Psi_{1}\left(k^{\prime}-1\right) e\left(k^{\prime}\right)+\frac{1}{b_{1}^{\prime}} \Psi_{2} \sum_{i=1}^{N} t_{i} c\left(k^{\prime}-i+1\right)\right) .
$$


Then,

$$
\|x(k)\| \leq \rho_{1} \sigma_{0}^{k-k_{0}}\left\|x\left(k_{0}\right)\right\|+\sum_{k^{\prime}=k_{0}}^{k} \rho_{1} \sigma_{0}^{k-k^{\prime}}\left(\rho_{2}+\rho_{3}\left|e\left(k^{\prime}\right)\right|\right)
$$

for some positive real constants $\rho_{i}, i \in\{1,2,3\}$, since $\Psi_{1}(k)$ is uniformly bounded and provided that the sequence $\{c(k)\}$ is bounded. From (3.16) and (4.1), it follows that $e(k)=$ $e^{0}(k)+\left(\widehat{\theta}^{0}(k-1)-\widehat{\theta}(k-1)\right)^{T} \widehat{\varphi}(k-1)$, and then

$$
|e(k)| \leq\left|e^{0}(k)\right|+\ell(k) \delta\|\widehat{\varphi}(k-1)\|
$$

where $\ell(k)$ denotes the finite number of times that the instruction while in the Step 2 of the estimates modification algorithm is executed at the current sampling time $k T$. By substituting (B.4) in (B.3), one obtains

$$
\|x(k)\| \leq \rho_{4}+\sum_{k^{\prime}=k_{0}}^{k} \rho_{5} \sigma_{0}^{k-k^{\prime}}\left(\left|e^{0}\left(k^{\prime}\right)\right|+\ell\left(k^{\prime}\right) \delta\left\|\widehat{\varphi}\left(k^{\prime}-1\right)\right\|\right),
$$

for some positive real constants $\rho_{4}$ and $\rho_{5}$ from the boundedness of $x\left(k_{0}\right)$. By using that $\widehat{\varphi}_{n}(k-$ $1)=\widehat{\varphi}(k-1) /(1+\|\widehat{\varphi}(k-1)\|)$ and $e_{n}^{0}(k)=e^{0}(k) /(1+\|\widehat{\varphi}(k-1)\|)$, it follows that

$$
\begin{aligned}
\|x(k)\| & \leq \rho_{4}+\sum_{k^{\prime}=k_{0}}^{k} \rho_{5} \sigma_{0}^{k-k^{\prime}}\left(\left|e_{n}^{0}\left(k^{\prime}\right)\right|+\ell\left(k^{\prime}\right) \delta\left\|\widehat{\varphi}_{n}\left(k^{\prime}-1\right)\right\|\right)\left(1+\left\|\widehat{\varphi}\left(k^{\prime}-1\right)\right\|\right) \\
& \leq \rho_{4}+\sum_{k^{\prime}=k_{0}}^{k} \rho_{6} \sigma_{0}^{k-k^{\prime}} e_{a n}^{0}\left(k^{\prime}\right)\left(1+\left\|\widehat{\varphi}\left(k^{\prime}-1\right)\right\|\right)
\end{aligned}
$$

for some positive real constant $\rho_{6}$ and, also, by taking into account that $\left(\left|e_{n}^{0}(k)\right|+\ell(k) \delta \| \widehat{\varphi}_{n}(k-\right.$ 1)\|) $\leq \rho^{\prime}\left(\left(e_{n}^{0}(k)\right)^{2}+\widehat{\varphi}_{n}^{T}(k-1) P^{2}(k-1) \widehat{\varphi}_{n}(k-1)\right)^{1 / 2}=\rho^{\prime} e_{a n}^{0}(k)$ for some positive real constant $\rho^{\prime}$. Moreover, from (B.6),

$$
\begin{aligned}
\|x(k)\| \leq & \rho_{4}+\sum_{k^{\prime}=k_{0}}^{k} \rho_{6} \sigma_{0}^{k-k^{\prime}} \mu\left(1+\frac{\widehat{\varphi}_{n}^{T}\left(k^{\prime}-1\right) P\left(k^{\prime}-1\right) \widehat{\varphi}_{n}\left(k^{\prime}-1\right)}{\gamma\left(k^{\prime}\right)}\right)^{1 / 2} \eta_{T}\left(k^{\prime}\right) \\
& +\sum_{k^{\prime}=k_{0}}^{k} \rho_{6} \sigma_{0}^{k-k^{\prime}} f\left(k^{\prime}\right)\left(1+\left\|\widehat{\varphi}\left(k^{\prime}-1\right)\right\|\right)
\end{aligned}
$$


where $e_{a n}^{0}\left(k^{\prime}\right)$ has been split into the two additive terms $f\left(k^{\prime}\right)$ and $e_{a n}^{0}\left(k^{\prime}\right)-f\left(k^{\prime}\right) \leq \mu \bar{\eta}_{a n}\left(k^{\prime}\right)$. Then,

$$
\begin{aligned}
\|x(k)\| \leq & \rho_{7}+\frac{\rho_{6} \mu v_{3}}{1-\sigma_{0}} \operatorname{Sup}_{k_{0} \leq k^{\prime} \leq k}\left\{\left(1+\frac{\widehat{\varphi}_{n}^{T}\left(k^{\prime}-1\right) P\left(k^{\prime}-1\right) \widehat{\varphi}_{n}\left(k^{\prime}-1\right)}{\gamma\left(k^{\prime}\right)}\right)^{1 / 2}\right\} \operatorname{Sup}_{k_{0} \leq k^{\prime} \leq k}\left\{\left\|x\left(k^{\prime}\right)\right\|\right\} \\
& +\sum_{k^{\prime}=k_{0}}^{k} \rho_{6} \sigma_{0}^{k-k^{\prime}} f\left(k^{\prime}\right)\left(1+\left\|\widehat{\varphi}\left(k^{\prime}-1\right)\right\|\right)
\end{aligned}
$$

for some positive constant $\rho_{7}$ by taking into account that $\bar{\eta}_{T}(k)=v_{3}\|x(k-1)\|+v_{4}$ (see Remark 3.1(iii)). Furthermore, the right-hand side of (B.8) is monotonic nondecreasing in $k$. Then, for $k \gg k_{0}$, it follows that

$$
\|x(k)\| \leq \rho_{8}+\sum_{k^{\prime}=k_{0}}^{k} \rho_{9} \sigma_{0}^{k-k^{\prime}} f\left(k^{\prime}\right)\left(1+\left\|\widehat{\varphi}\left(k^{\prime}-1\right)\right\|\right)
$$

provided that $v_{3}<\left(\left(1-\sigma_{0}\right) / \rho_{6} \mu\right)\left(\operatorname{Sup}_{k_{0} \leq k^{\prime} \leq k}\left\{\left(1+\widehat{\varphi}_{n}^{T}\left(k^{\prime}-1\right) P\left(k^{\prime}-1\right) \widehat{\varphi}_{n}\left(k^{\prime}-1\right) / \gamma\left(k^{\prime}\right)\right)^{1 / 2}\right\}\right)^{-1}$, for some positive constants $\rho_{8}$ and $\rho_{9}$. By taking into account that $\|\widehat{g}(k)\|$ is bounded for all $k \in \mathbb{Z}_{0}^{+}$, then

$$
\|\widehat{\varphi}(k-1)\| \leq \rho_{10}+\rho_{11} \operatorname{Sup}_{k_{0} \leq k^{\prime} \leq k}\left\{\left\|x\left(k^{\prime}-1\right)\right\|\right\}
$$

for some positive constants $\rho_{10}$ and $\rho_{11}$. By substituting (B.10) in (B.9) and by taking into account that the right-hand side of (B.9) is monotonic nondecreasing in $k$, it follows that,

$$
\operatorname{Sup}_{k_{0} \leq k^{\prime} \leq k}\left\{\left\|x\left(k^{\prime}\right)\right\|\right\} \leq \rho_{12}+\sum_{k^{\prime}=k_{0}}^{k} \rho_{13} f\left(k^{\prime}\right) \operatorname{Sup}_{k_{0} \leq k^{\prime \prime} \leq k^{\prime}}\left\{\left\|x\left(k^{\prime \prime}-1\right)\right\|\right\}
$$

for some positive constants $\rho_{12}$ and $\rho_{13}$, where the boundedness of $f(k)$ has been used, see Lemma 3.2. The use of Gronwall's Lemma [24] in (B.11) leads to

$$
\operatorname{Sup}_{k_{0} \leq k^{\prime} \leq k}\left\{\left\|x\left(k^{\prime}\right)\right\|^{2}\right\} \leq \rho_{14}+\sum_{i=k_{0}}^{k}\left(\prod_{i<j<k}\left(1+\rho_{15} f^{2}(j)\right)\right) \rho_{14} \rho_{15} f^{2}(i)<\infty
$$

for some positive constants $\rho_{14}$ and $\rho_{15}$. It implies that the sequence $\|x(k)\|$ is bounded for all $k \in \mathbb{Z}^{+}$provided that the initial condition is bounded. Then, the sequences $\{u(k)\}$ and $\{y(k)\}$ are also bounded.

(ii) The adaptive control algorithm ensures that there are not finite escape times, and then the boundedness of the sequences $\{u(k)\}$ and $\{y(k)\}$ guarantees that of the continuoustime signals from continuity arguments of the solutions of differential equations. 


\section{Acknowledgments}

The authors are very grateful to MCYT for its support through Grants nos. DPI2006-00714 and DPI 2009-07197. They are also very grateful to the reviewers for their useful comments, which have helped to improve the previous versions of the manuscript.

\section{References}

[1] P. A. Ioannou and J. Sun, Robust Adaptive Control, Prentice-Hall, Englewood Cliffs, NJ, USA, 1996.

[2] S. Alonso-Quesada and M. De la Sen, "Robust adaptive control of discrete nominally stabilizable plants," Applied Mathematics and Computation, vol. 150, no. 2, pp. 555-583, 2004.

[3] S. Alonso-Quesada and M. De la Sen, "Robust adaptive stabilizer for linear systems with imperfectly known point delays using a multi-estimation model," Dynamics of Continuous, Discrete E Impulsive Systems. Series B, vol. 15, no. 5, pp. 683-708, 2008.

[4] M. De la Sen and A. Ibeas, "On the global asymptotic stability of switched linear time-varying systems with constant point delays," Discrete Dynamics in Nature and Society, vol. 2008, Article ID 231710, 31 pages, 2008.

[5] M. De la Sen and A. Ibeas, "Stability results of a class of hybrid systems under switched continuoustime and discrete-time control," Discrete Dynamics in Nature and Society, vol. 2009, Article ID 315713, 28 pages, 2009.

[6] A. Ibeas, M. De la Sen, and S. Alonso-Quesada, "Stable multi-estimation model for single-input single-output discrete adaptive control systems," International Journal of Systems Science, vol. 35, no. 8, pp. 479-501, 2004.

[7] M. De la Sen and S. Alonso-Quesada, "Model matching via multirate sampling with fast sampled input guaranteeing the stability of the plant zeros: extensions to adaptive control," IET Control Theory E Applications, vol. 1, no. 1, pp. 210-225, 2007.

[8] S. Liang and M. Ishitobi, "Properties of zeros of discretised system using multirate input and hold," IEE Proceedings: Control Theory and Applications, vol. 151, no. 2, pp. 180-184, 2004.

[9] S. Liang, M. Ishitobi, and Q. Zhu, "Improvement of stability of zeros in discrete-time multivariable systems using fractional-order hold," International Journal of Control, vol. 76, no. 17, pp. 1699-1711, 2003.

[10] B. Iričanin and S. Stević, "On some rational difference equations," Ars Combinatoria, vol. 92, pp. 67-72, 2009.

[11] S. Stević, "On a generalized max-type difference equation from automatic control theory," Nonlinear Analysis: Theory, Methods E Applications, vol. 72, no. 3-4, pp. 1841-1849, 2010.

[12] K. S. Narendra and A. M. Annaswamy, Stable Adaptive Systems, Prentice-Hall, Englewood Cliffs, NJ, USA, 1989.

[13] P. A. Ioannou and Datta, "Robust adaptive control: a unified approach," Proceedings of the IEEE, vol. 79, no. 12, pp. 1736-1768, 1991.

[14] R. H. Middleton, G. C. Goodwin, D. J. Hill, and D. Q. Mayne, “Design issues in adaptive control," IEEE Transactions on Automatic Control, vol. 33, no. 1, pp. 50-58, 1988.

[15] S. Alonso-Quesada and M. De la Sen, "Robust adaptive control with multiple estimation models for stabilization of a class of non-inversely stable time-varying plants," Asian Journal of Control, vol. 6, no. 1, pp. 59-73, 2004.

[16] K. G. Arvanitis, "An algorithm for adaptive pole placement control of linear systems based on generalized sampled-data hold functions," Journal of the Franklin Institute, vol. 336, no. 3, pp. 503 521, 1999.

[17] M. J. Błachuta, “On approximate pulse transfer functions," IEEE Transactions on Automatic Control, vol. 44, no. 11, pp. 2062-2067, 1999.

[18] L. Chen and L. Chen, "Permanence of a discrete periodic Volterra model with mutual interference," Discrete Dynamics in Nature and Society, vol. 20089, Article ID 205481, 9 pages, 2009.

[19] Y.-H. Fan and L.-L. Wang, "Permanence for a discrete model with feedback control and delay," Discrete Dynamics in Nature and Society, vol. 2008, Article ID 945109, 8 pages, 2008.

[20] C. Wei and L. Chen, "A delayed epidemic model with pulse vaccination," Discrete Dynamics in Nature and Society, vol. 2008, Article ID 746951, 12 pages, 2008.

[21] H. R. Karimi, M. Zapateiro, and N. Luo, "New delay-dependent stability criteria for uncertain neutral 
systems with mixed time-varying delays and nonlinear perturbations," Mathematical Problems in Engineering, vol. 2009, Article ID 759248, 22 pages, 2009.

[22] G. Tao and P. A. Ioannou, "Model reference adaptive control for plants with unknown relative degree," IEEE Transactions on Automatic Control, vol. 38, no. 6, pp. 976-982, 1993.

[23] G. C. Goodwin and K. S. Sin, Adaptive Filtering Prediction and Control, Prentice-Hall, Englewood Cliffs, NJ, USA, 1984.

[24] C. A. Desoer and M. Vidyasagar, Feedback Systems: Input-Output Properties, Academic Press, New York, NY, USA, 1975. 


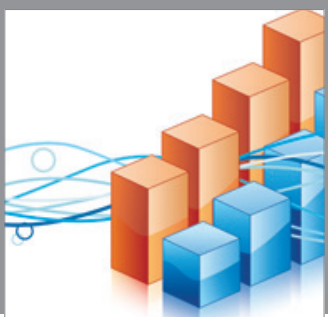

Advances in

Operations Research

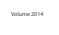

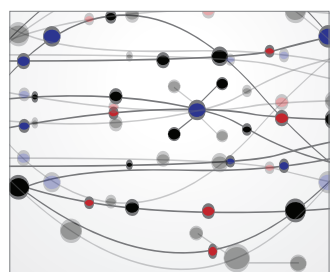

\section{The Scientific} World Journal
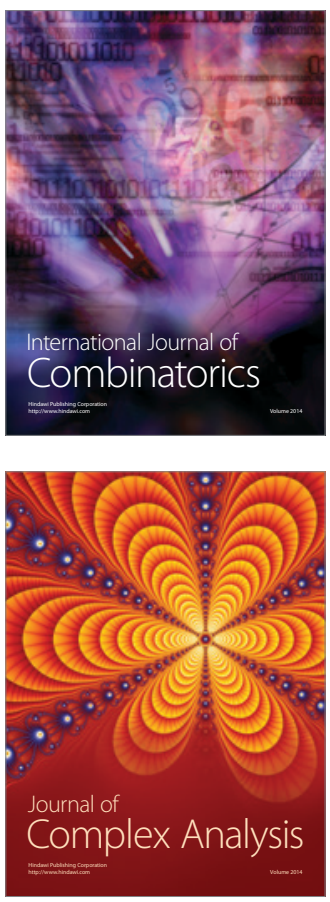

International Journal of

Mathematics and

Mathematical

Sciences
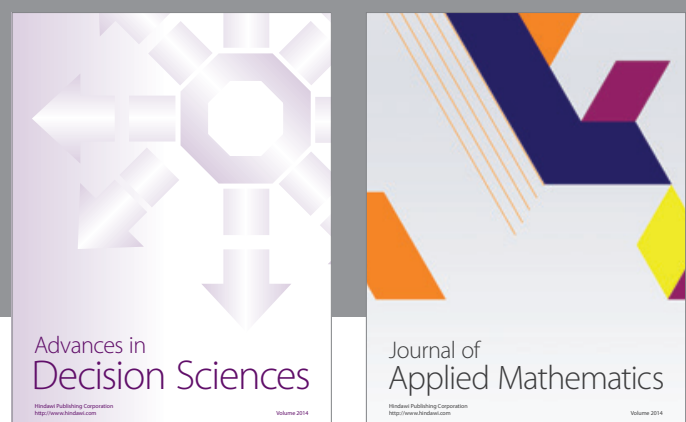

Journal of

Applied Mathematics
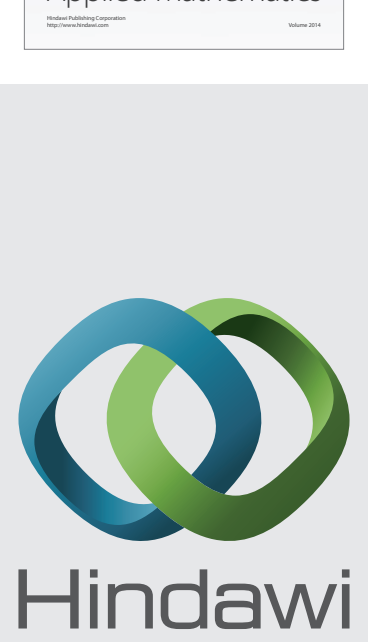

Submit your manuscripts at http://www.hindawi.com
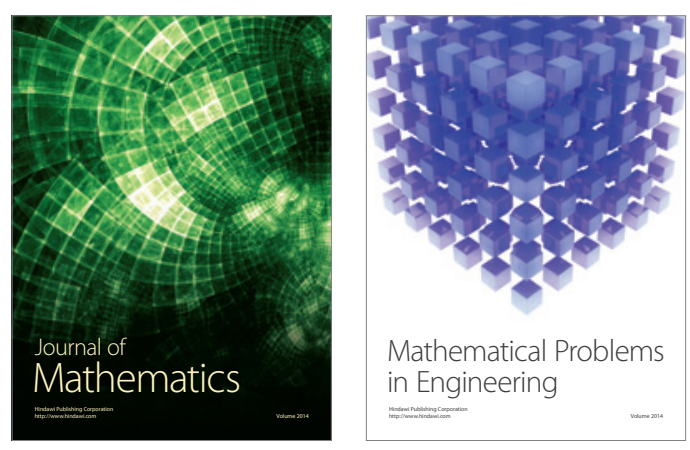

Mathematical Problems in Engineering
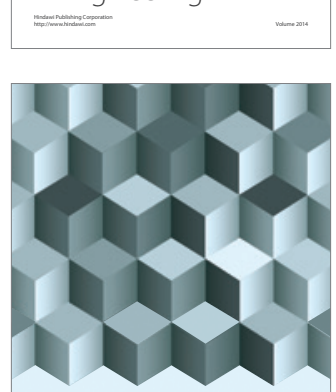

Journal of

Function Spaces
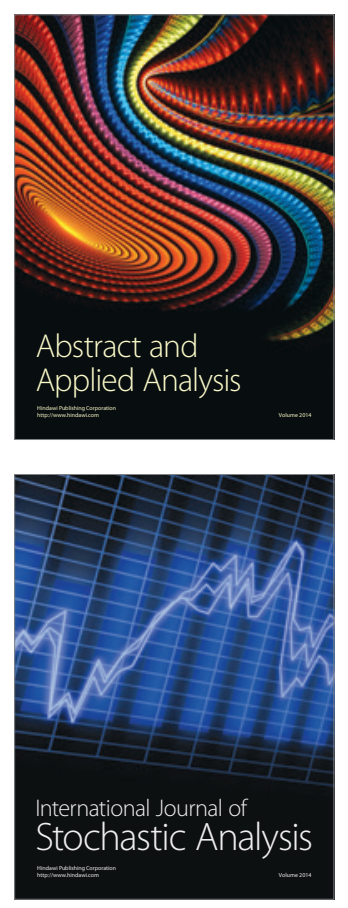

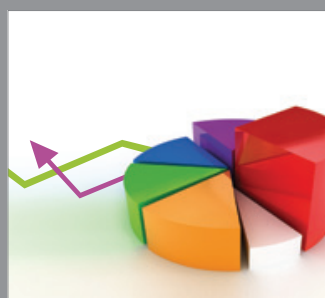

ournal of

Probability and Statistics

Promensencen
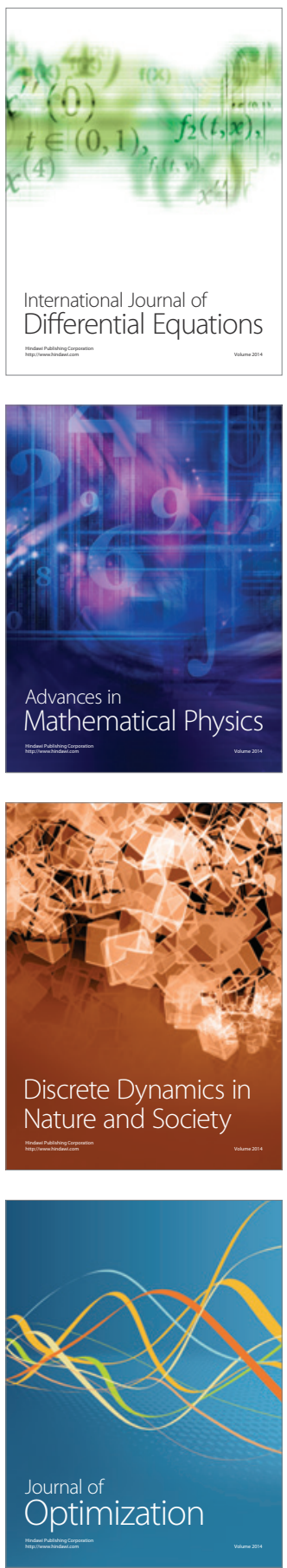